\title{
Characters of neoantigens in cancer immunotherapy
}

2 Peng Bai ${ }^{1}$, Yongzheng $\mathrm{Li}^{1}$, Qiuping Zhou ${ }^{1}$, Jiaqi Xia ${ }^{1}$, Min $\mathrm{Wu}^{2}$, Hexiang Deng ${ }^{3}$, John W. Kappler, ${ }^{4,5,6,7}$,

51 State Key Laboratory of Virology, Hubei Key Laboratory of Cell Homeostasis, College of Life Sciences,

6 Wuhan University, Wuhan, 430072, Hubei, China

72 Hubei Key Laboratory of Cell Homeostasis, College of Life Sciences, Wuhan University, Wuhan,

$8 \quad 430072$, Hubei, China

93 Key Laboratory of Biomedical Polymers, Ministry of Education, the Institute for Advanced Studies,

7 Structural Biology and Biochemistry program, University of Colorado Anschutz Medical Campus, Aurora, CO 80045, USA 


\section{Abstract}

Cancer mutations lead to the production of mutated proteins from which can be processed and presented to $\mathrm{CD}^{+} \mathrm{T}$ cells as neoantigens. Despite the promise of immunotherapy targeting neoantigens, which mutant peptides are likely to induce immune responses remain elusive. Here, we determined two sets of MHC-I bound neoantigen structures and performed bioinformatics investigation based on neoantigen data. Structural and bioinformatic analyses reveal that some types of mutations in immunogenic neoantigens are preferentially selected. We also developed a strategy to expand the usage of a known neoantigen by scanning potential MHC alleles. Overall, our results will extend the ability to discover neoantigens that may be useful for cancer immunotherapy.

\section{Introduction}

The immune system can sometimes recognize and destroy cancer cells $(1)$. $\mathrm{CD}^{+} \mathrm{T}$ cells have been demonstrated to be a major component in this process by both recognizing and destroying the target cells(2-5). For T cells to react with tumor cells two components are required, major histocompatibility complex class I and possibly class II proteins (MHC-I, MHC-II) on the tumor cells and peptides which processed by the tumors bound to these $\mathrm{MHC}$ proteins. $\mathrm{CD} 8^{+} \mathrm{T}$ cells can then recognize the combination of MHCI and tumor derived peptides on the cancer cells. Thus, it is crucial to identify effective tumor antigenic peptides with their relevant MHCs.

During the last 25 years, great efforts have been made to identify tumor antigens(6). These tumor antigens can be classified into two broad categories, self-antigens which are seldom expressed in normal tissue or expressed at much higher levels on the cancer cell versus normal tissues (like NYESO-1, MART-1) and non-self-antigens called neoantigens which are derived from somatic mutations in the tumor(7). The neoantigens are not presented by normal tissues, hence the immune system is not tolerant to them and views them, correctly, as foreign antigens and responds appropriately $(8,9)$.

Recently, next-generation sequencing (NGS) provide formidable identification of tumor-specific nonsynonymous mutations, frameshift mutations, and gene rearrangements, which have driven clinical immunotherapeutic methods such as vaccination, adoptive cell transfer (ACT) and blockade of the proteins that inhibit $\mathrm{T}$ cell activation, have demonstrated that immunotherapy targeting tumor neoantigens can induce objective clinical responses in many patients with metastatic cancers(10-13). It 
appears that $\mathrm{T}$ cell targeting of tumor neoantigens might represent the "final common pathway" to treat cancer(14). However, neoantigen discovery and validation remains a daunting problem. Classical cDNA expressing screening methods to identify tumor neoantigens without knowledge of the properties needed for a mutated peptide to be a successful immunotherapeutic is not efficient(15). To solve this problem, recently, a study identified mouse neoantigens by combination of NGS and prediction algorithms for antigen presentation by $\mathrm{MHCI}(16)$. In brief, they compared mouse tumor and normal DNA for identifying non-synonymous mutations and used peptide/MHCI binding prediction algorithms to find mutated peptides which could bind MHC to be potential effective neoantigens. These advances of discovering neoantigens bring us a step closer to personalize immunotherapy.

Indeed, neoantigen prediction lighten the burden of immunogenicity testing by reducing the number of candidate peptides(17-27). Nevertheless these algorithms are not robust enough to identify immunogenic neoantigens accurately $(14,28)$. There are several reasons for this. Firstly, many of these algorithms do not consider the likelihood that the peptide in question will be properly processed and transported to the endoplasmic reticulum, where they bind MHCI. This problem can be solved by adding other methods, such as NetChop(29) (for antigen processing prediction) and NetCTL(30) (for peptide transport prediction) to the prediction process. Secondly, the MHC binding prediction methods based on artificial neural network (ANN) models need a lot of data ,such as those available in NetMHC 4.0, for training(31). However, large amounts of these input data are lacking for many human MHC alleles. Also, in some cases, most peptides in the training data were of non-tumor origin such as cultured cell lines and microbial foreign peptides. Thus, it is unclear whether these non-self peptidome data accurately represent the profile of mutated-self neoantigens and elicit $\mathrm{T}$ cell response(32). Thirdly, among neoantigens binding MHC actually, only a small portion of them are immunogenic and have therapeutic effects(33). Thus, it is crucial to investigate the details of neoantigen immunogenicity and to improve the performance of in silico neoantigen prediction algorithm.

Recently, Tran et al. reported a remarkable case of a patient whose metastatic colorectal cancer regressed after infusion of HLA-C*08:02-restricted tumor-infiltrating lymphocytes (TILs) targeting KRAS G12D neoantigens. Kras encodes a small GTPase that mediates downstream signaling from growth factor receptors, and it is one of the most infamous driver genes led to oncogenesis. The most common KRAS mutation is a gain-of-function substitution of the glycine to aspartic acid at codon 12 (KRAS G12D), a change that is often present in colorectal or pancreatic cancer(34-36). The KRAS 
G12D mutant 9mer peptide GADGVGKSA and 10mer peptide GADGVGKSAL can both stimulate autologous $\mathrm{T}$ cells in the context of HLA-C*08:02, while their wild type counterparts cannot(23). Adoptive cell transfer (ACT) of T cells specific for the single mutant neoantigen led to tumor disappearance in patient. Thus, the KRAS G12D neoantigens have been acknowledged to be useful and typical neoantigen that might be used as a model to illustrate the secret of therapeutic tumor neoantigens.

Here we took structural analyses of several neoantigens with their relevant MHCs, combined with a broad examination of immunotherapeutic and non-immunogenic tumor antigens. We concluded that a universal type of tumor neoantigen peptide mutations from non-preferential residues to preferential residues with their relevant MHCs at anchor positions is unexpectedly a good indicator to predict neoantigen immunogenicity. Combined with other findings about effective neoantigens we thus propose an optimized strategy for neoantigen identification.

\section{Results}

\section{The structures of HLA-C*08:02 in complex with KRAS G12D neoantigens}

As described above, Tran and colleagues reported a clinical case which showed substantial tumor regression after adoptive $\mathrm{T}$ cell transfer of $\mathrm{T}$ cells specific for HLA-C*08:02 bound to the mutant KRAS G12D 9mer and 10mer peptides(23). HLA-C*08:02 is expressed in approximately $8 \%$ of whites(23) and 7\% of African Americans(37) in the U.S. The KRAS G12D mutation is most common in pancreatic and colorectal cancers, being present in approximately $45 \%$ and $13 \%$ of these two tumor types, respectively(38). Thus, overall about $2.3 \%$ patients with colorectal cancer share the HLAC*08:02 allele and KRAS G12D mutation (Supplementary Table 1) and these results suggest that thousands of patients per year in the United States alone could potentially be eligible for the therapy targeting HLA-C*08:02-restricted KRAS G12D neoantigens.

With this in mind we decided it would be useful to know the structure of HLA-C*08:02 in complex with four peptides: wild type KRAS G12D 9mer GAGGVGKSA (wt9m); wild type KRAS G12D 10mer peptide GAGGVGKSAL (wt10m); mutant KRAS G12D 9mer peptide GADGVGKSA (mut9m, mutation site is indicated with the amino acid underlined) and mutant KRAS G12D 10mer peptide 
GADGVGKSAL (mut10m). HLA-C*08:02 heavy chain and light chain $\beta 2$-microglobulin $(\beta 2 \mathrm{M})$ were expressed in E. coli as inclusion bodies and refolded with peptides. C08-mut9m and C08-mut10m complex were successfully refolded and detected on SDS-PAGE gels. Unfortunately, we failed to refold C08-wt9m complex and C08-wt10mer complex even with a ten-fold increase in concentration of the peptide (data not shown). This result suggested that the glycine to aspartic acid mutation of these neoantigens is important to maintain the pMHC stability. However, the artificial neural network (ANN) based peptide binding algorithm NetMHC4.0(39) predicted that binding affinity of KRAS G12D mutated neoantigens (both 9mer and 10mer) and their wild type counterparts for HLA-C*08:02 would all be very low (less than $15,000 \mathrm{nM}$, Supplementary Table 2). This could be due to relatively less training data of HLA-C*08:02.

We solved the structures at 2.4 angstroms $(\AA)$ for the C08-mut9m complex and $1.9 \AA$ for the C08mut10m complex (Supplementary Table 3 ). The high-quality resolution and unbiased electron density at the region involving the peptides (Fig.1a, b) allowed reliable model building. The C08-mut9m complex and the C08-mut10m complex shown highly conserved conformation except the peptide region (Fig.1 c, d).

Peptide amino acid binding motif of HLA-C*08:02 shows that smaller residues such as alanine and serine are preferably selected at P1 and P2 position (Fig.1e). This preference is due to the narrow cleft formed by several C*08:02 aromatic residues (Tyr7, Phe33, Tyr67, Tyr99, Tyr59, Tyr171, Tyr159 and Trp167), which limit the size of the P1 and P2 side chains that can bind at that site (Fig.1f). C08mut9m and C08-mut10m are relatively similar at the position $1(\mathrm{P} 1)$ and position $2(\mathrm{P} 2)$ of the peptides. In C08-mut9m and C08-mut10m the N-terminal P1G (Glycine at position 1) residue of the peptides form hydrogen bonds with Tyr7, Tyr159 and Tyr171 and the P2A residues interact with Glu63 and Lys66 (Fig. 2a).

Differences appeared at the P3-P $\Omega$ (P $\Omega$ : C-terminal position of peptide) regions of the peptides. Although P3D in C08-mut9m and C08-mut10m both act as anchor residues, the side chain carboxyl group of P3D forms an intra-chain hydrogen bond with P4G in mut9m peptide, while this hydrogen bond is absent in the mut $10 \mathrm{~m}$ peptide structure. The hydrogen bond in the mut $9 \mathrm{~m}$ structure pulls down the arch of the 9 mer peptide, causing the region of the 9mer peptide that is accessible to TCRs to differ from that of the 10 mer peptide. The greatest difference between the two structures is apparent in the 
center of the peptide (P4-P7), where each complex differs in the contacts it offers for TCR recognition.

The P8S has different orientations in the two structures and acts as an auxiliary anchor in C08-mut10m. Differences were also observed at the C-termini of the peptides (Fig. 1c; Fig. 2b, c). The P9A of C08mut9m forms hydrogen bonds with Ser77, Asn80, Tyr84, Thr143 and Lys146, and acts as an anchor residue (Fig. 2b). The P9A of C08-mut10m is totally different from the P9A of C08-mut9m with the side chain up. However, the P10L of C08-mut10m has similar orientation towards HLA-C*08:02 and plays a similar role as P9A in C08-mut9m (Fig. 1c; Fig. 2b, c).

\section{HLA-C*08:02 presents KRAS G12D neoantigens with an unconventional MHC anchor motif}

Typically, peptides always use anchor positions at $\mathrm{P} 2$ and $\mathrm{P} \Omega$ to bind the $\mathrm{B}$ and $\mathrm{F}$ pockets respectively of HLA class I alleles. The structures of the C08-mut9m and C08-mut10m reveal that G12D 9mer and 10mer peptides all use unconventional $\mathrm{P} 3$ and $\mathrm{P} \Omega$ residues for anchors to form stable complexes with HLA-C*08:02 (Fig.1c; Fig. 2b, c) thus differing from the canonical HLA Class I binding pattern (using $\mathrm{P} 2$ and $\mathrm{P} \Omega$ residues for anchors). In this case, the B pocket of HLA-C*08:02 binds the P3D anchor residue via interactions with Arg97 and Arg156. Although the 9mer and 10mer peptides occupy the same F pocket with their $\mathrm{P} \Omega$ residues as anchors, the P10L side chain from the 10 mer peptide is buried more deeply than P9A in the 9mer structure (Fig.1c; Fig. 2b, c). Also, instead of the upward residue, P8S, in 9mer peptide, the 10mer peptide uses P8S as an auxiliary anchor, binding the HLA protein via hydrogen bonds with Glu152 and Arg156. These interactions squeeze the P4 to P7 residues together with residue P3D (Fig. 2c). Also, together with P3D, the existence of auxiliary anchor residue P8S arches the P4-P7 residues of the 10mer peptide to a higher position than that in the 9mer peptide (Fig. $2 \mathrm{~b}, \mathrm{c})$. This alteration likely increases the binding affinity of the 10 mer peptide with HLA and changes the pHLA binding surface for the T cell receptor (TCR).

KRAS G12D 9mer and 10mer peptides were previously reported to stimulate different T-cell clones with different TCR variable chains(10). Our structural analyses are in line with such a report since C08-mut9m and C08-mut10m provide different binding surfaces for T cell recognition (Fig.1b; Fig. 2b, c). Furthermore, both wild type counterparts of 9mer and 10mer peptides failed to refold with HLA$C^{*} 08: 02$, which means that the G12D mutation is important for forming the pMHC complex. Structural analysis shows that G12D mutations of the KRAS neoantigens act as anchors and stabilize contacts 
with the MHC. Thus, in this case, the KRAS mutation shows the likelihood that anchor mutated neoantigens will exist to stimulate $\mathrm{T}$ cells.

\section{Structural basis of non-therapeutic mouse neoantigen DPAGT1 V213L in complex with H-2 Kb}

Having verified that the P3D residue of KRAS G12D neoantigens plays an important role in formation of the pMHC complex, we studied whether other cancer neopeptides with anchor mutations had similar properties. One such neopeptide, which is not immunotherapeutic, is the mutation of the $\mathrm{C}$ terminal amino acid anchor residue of the mouse mutant DPAGT1 V213L peptide(7). To study this we solved the structure of mouse MHCI H-2 Kb in complex with mutant DPAGT1 V213L 8mer peptide (SIIVFNLV, mutation site is indicated with the amino acid underlined) and the wild type 8mer counterpart (SIIVFNLL) (Supplementary Table 3). The last residue of the peptide, a valine in the wild type 8 mer peptide (wt $8 \mathrm{mV}$ ) was replaced by leucine in the mutant 8 mer peptide (mut $8 \mathrm{~mL}$ ). Soluble complexes of $\mathrm{Kb}$-wt $8 \mathrm{mV}$ and $\mathrm{Kb}$-mut $8 \mathrm{~mL}$ were separately expressed, refolded and purified for crystallization trials. Crystal diffraction data of $\mathrm{Kb}-\mathrm{wt} 8 \mathrm{mV}$ and $\mathrm{Kb}-\mathrm{mut} 8 \mathrm{~mL}$ are processed to $2.4 \AA$ and $2.5 \AA$ resolution respectively (Supplementary Table 3 ) and provides unambiguous electron density for each peptide (Supplementary Fig.1a).

The overall structure of the $\mathrm{Kb}$-wt $8 \mathrm{mV}$ complex closely resembles that of $\mathrm{Kb}$-mut8mL with the exception of a slight difference at $\mathrm{P} \Omega(\mathrm{P} 8)$ (Supplementary Fig.1b). The C-terminal $\mathrm{P} \Omega$ residue acts as an anchor in both the $\mathrm{Kb}-\mathrm{wt} 8 \mathrm{mV}$ and $\mathrm{Kb}$-mut8mL complexes (Supplementary Fig.1b). Moreover, both $P \Omega$ valine and leucine were preferably selected by $\mathrm{H}-2 \mathrm{~Kb}$ in a peptide library analysis (Supplementary Fig.1c). Both of the P $\Omega$ residues formed hydrogen bonds with Asp77, Tyr84, Thr143 and Lys146 of Kb but the side chain of leucine in the mut $8 \mathrm{~mL}$ peptide is insert more deeply into $\mathrm{Kb}$ than valine in wt $8 \mathrm{mV}$ peptide because of longer side chain of the Leucine residue. Thus, P8L is able to make Van der Waals contact with Tyr116 on the floor of the F pocket (Supplementary Fig.1d, e). These alterations did not significantly affect the formation of peptide/Kb complex and change the TCR binding surface, suggesting that, as far as $\mathrm{T}$ cell recognition is concerned the mut $8 \mathrm{~mL}$ neoantigen would be treated as a "self-peptide", thus minimizing mut $8 \mathrm{~mL}$ neoantigen immunogenicity in vivo. All these indicate the wild type and mutant DPAGT1 V213L peptides interact with $\mathrm{Kb}$ in a similar way, thus the mutation might provide neither a higher concentration of peptide/ $\mathrm{Kb}$ on the cell surface nor a TCR interface that differs. This is unlike the case of the KRAS G12D mutant peptides which appear to interact more 
195 intensively with HLA, via the mutant acid residue P3D interaction with basic residues in the B pocket

196 of $C^{*} 08: 02$, versus than the wild type counterpart.

197 Taken together, all these suggest that whether wild type or mutant amino acids at anchor positions

198 interacted differently with relevant HLA alleles might be key to neoantigen immunogenicity.

\section{Curated datasets of human immunogenic neoantigens from publications}

201 To extend these analyses, bioinformatic studies were performed on all available immunogenic and nonimmunogenic neoantigen peptide/MHC complexes. We manually gathered immunogenic neoantigens associated with various tumor types from recently published papers. This immunogenic neoantigen dataset (IND) encompassed 128 neoepitopes (neoantigens and their relevant HLAs) from 15 types of human cancer. Each of them was well tested by at least tested in vitro T cell assays. Less well characterized neoantigens and the neoantigens without minimal length were not included. Each entry included information such as the cancer type, HLA allele type, gene name, peptide sequence and other characteristics which are important in cancer immunotherapy. Melanoma and non-small cell lung cancer contain higher mutational burdens and have been more extensively studied than others, thus nearly $80 \%$ entries in our dataset came from them.

211 We also gathered 11739 non-immunogenic neopeptides (peptides splice from mutant proteins) from 13 published papers termed raw non-immunogenic neopeptide dataset (RNND). All of these mutant peptides were tested in in vitro $\mathrm{T}$ cell assays or clinical trials and did not elicit effective immune responses. Notably, the vast majority of them were not the minimal peptides known to induce $\mathrm{CD} 8^{+} \mathrm{T}$ cells response (most of them are 25 mer peptides). Thus, we use the prediction algorithm NetMHCpan 4.0(40) to predict the conventional minimal length (9mers and 10mers) non-immunogenic neopeptides with relevant HLAs under a cut-off IC50=500 nM. This dataset was termed non-immunogenic neopeptide dataset (NND). This dataset contains 2883 putative entries. It turns out that many neoantigens predicted to bind patients' HLAs very well are experimentally tested to be nonimmunogenic and do not have the ability to evoke effective immune response. This fact compromised

221 the usage of predicting immunogenic neoantigen through prediction of neoantigen binding to patients' HLAs. 


\section{Evident differences of mutation distribution between immunogenic and non-immunogenic} peptides

It is thought that immunogenic neoantigens would bind MHC well and the mutations in the immunogenic neoantigens could increase the affinity for binding MHC. With our immunogenic neoantigen data collected, we preformed binding prediction (NetMHCpan 4.0) of wild type and mutant peptides in IND and showed the distribution of affinity ratio between them (Fig.3a). About $25.5 \%$ of immunogenic neoantigens shows the predicted binding affinity above 500nM and about $24 \%$ do not exhibit increased prediction binding affinity (WT/MUT affinity ratio $<1$ ). Thus, more characters of immunogenic neoantigens which could help immunogenic neoantigen predictions are needed.

It is suspected that the position in the peptides of mutated amino acids could affects the immunogenicity of neoantigens. To further investigate the mutation distribution at each peptide position between immunogenic and non-immunogenic data, we calculated the mutation occurrence frequency at each position of 9 mer and 10mer peptides. Interestingly, both immunogenic 9mer and 10mer neoantigens show frequency fluctuation at each position with their mutations. The fluctuation of non-immunogenic 9 mer and 10 mer neoantigens seems not as high as those from immunogenic dataset (Fig. 3b; Supplementary Fig. 2). However the difference is not reaching significance probably due to the limited size of so far available immunogenic data. Mutations in 9mer neoantigens were more frequent at P2, P4, P5 and P8 position and relatively lower frequency at P3 and P7 positions, relative to non-immunogenic 9mer data (Fig. 3b). Conceptually, when bound to HLA, peptides usually use P2 and $\mathrm{P} \Omega$ as anchor residues and use P4, P5 and P8 to contact TCR. Other residues in the peptide may contact with MHC but usually contribute less to the affinity of the peptide-HLA interaction(41). Also to be taken into this account is the fact that our structural studies above also show that mutations at anchor positions can evoke effective immune responses. Thus, these observations enlightened us to classify the mutation based on whether it located on the anchor (contribute primary binding force to MHC), MHC contact (contact MHC but contribute much less for binding MHC) or TCR contact positions for further study.

To find out the mutation location (anchor, MHC contact position and TCR contact position) of each peptide in IND an NND respectively, a strategy was developed to define the mutations location of 
neoantigens by combining the SYFPEITHI database(42), antigen binding motif from NetMHCpan(40) and solved pMHC structures from Protein Data Bank (see Methods). We chose 9mer length of neoantigens to do these studies since 9 mer peptides are most frequent in both the IND and NND collections. Peptides bound to MHC alleles for which structure models were not available in PDB were excluded from these analyses.

The 9mer neoantigens (or neopeptides) with mutations from IND and NND were divided into three functional classes based on the following: anchor mutation, MHC contact position mutation or TCR contact position mutation (Fig. 3c). After calculating the frequency of each class, we found that the immunogenic neoantigens were significantly more likely than NND to express mutations at TCR contact region (Fig. 3d, p<0.05, Z-test) and less likely to include mutations at MHC contact regions (excluding those at anchor positions) (Fig. $3 \mathrm{~d}, \mathrm{p}<0.01$, Z-test). These results can partially support proposals of others that neopeptides with TCR contact position mutation will preferentially be immunogenic(7). However, the odds of mutations at the anchor positions were similar between INDs and NNDs, therefore the importance of anchor position mutations in immunogenicity is not clear (Fig. 3d). These results are consistent with our structural studies showed that the immunogenic neoantigen KRAS G12D and the non-immunogenic neopeptide DPAGT1 V213L can both present by MHC.

\section{Certain type of mutation change at anchor position could be a clear indicator to distinguish}

\section{immunogenic neoantigens and non-immunogenic neopeptides}

It is thought that the nature of mutated amino acids affects the neoantigen's immunogenicity. To find out if this is so, we calculated the frequency with which different amino acids appeared as the mutations in either IND or NND neoantigens. So far Leu and Tyr are likely to show up in the mutation position from immunogenic neoantigens (Supplementary Fig. 3). This could be due to the idea that mutation to Leu and Tyr might supply good contacts with MHC to stabilize the binding of neoantigens to MHC or good contacts with TCR to stimulate T cells. This is consistent with our previously studies that Tyr is important in TCR-peptide/MHC recognition(43-46).

Next, to check the similarity between wild type and the mutant amino acids of neoantigens and nonimmunogenic neopeptides, we defined similarity of those amino acids from IND and NND using the normalized BLOSUM62 substitution matrix(47). In this matrix, bigger normalized score represents 
more similarity between the wild type and the mutant peptides. For the situation of immunogenic data compare to non-immunogenic data, the peptides with immunogenicity did not shown significant difference between wild type and the mutant amino acids (Fig. 4a, $\mathrm{p}=0.2077$, student t-test). For the situation of anchor mutated peptides, the similarity of immunogenic neoantigens to their wild type counterparts turn out to be lower than the similarity of the non-immunogenic peptides (Fig. 4b, $\mathrm{p}=0.001$, student $\mathrm{t}$-test). For the situation of 9 mer peptides with TCR contact position mutation, similar result occurs between immunogenic and non-immunogenic data (Fig. 4c, p=0.019, student t-test). We did not test for mutations at MHC contact regions, because the available data was too limited so far. These results demonstrated that through the mutation position classification quantifiable amino acid differences between mutation and their wild type counterparts do indeed exist at anchor and TCR contact positions between immunogenic and non-immunogenic data.

To test whether the mutant peptides in IND are more preferentially presented by HLAs than wild type peptides, we generated amino acids half-bits of each HLA from P1 to P9 (we test 9mer peptides only due to their relatively abundant quantity) which present in IND, based on the binders collected from IEDB (see Methods). Then, merged bits of mutated neoantigens and their wild type peptides with their relevant HLAs were calculated respectively (Fig. 4d-f). To our knowledge, peptide with higher merge bits means preferentially presented by HLA. We first tested peptide merge bits in all 9mer neoantigens and their wild type counterparts. The higher merged were shown in mutant neoantigens compare to their wild type counterparts, which means immunogenic neoantigens more likely to be presented by their relevant HLAs (Fig. 4d, p=0.0088, student t-test). We next tested this character from the data of 9mer anchor mutated neoantigen and 9mer TCR contact position mutated neoantigen. Surprisingly, anchor mutated neoantigen showed significantly higher preference to be present by their relevant HLAs than their wild type counterparts (Fig. $4 \mathrm{e}, * * * * \mathrm{p}<0.0001$, student $\mathrm{t}$-test), while TCR contact position mutated data did not shown any significance (Fig. 4f, $\mathrm{p}=0.9358$, student $\mathrm{t}$-test). From above results, it is evident that mutations of immunogenic neoantigens in the anchor positions and in the TCR contact positions shows different characters and should be studied separately. Moreover, the mutations of immunogenic neoantigens in the anchor positions were needed for further studies. decided to find out how the mutations and wild type amino acids in immunogenic neoantigens were 
311 similar for immunogenic and non-immunogenic peptides. The cut-off for preferential amino acids was 312 applied to be above $10 \%$ (include $10 \%$ ) as described previouslly(48) based on the data from peptide 313 library (Supplementary Fig. 4). Neoantigen mutations changes from wild type to mutant at anchor 314 positions were divided into 4 types: non-preferential to non-preferential residues ( $\mathrm{N}$ to $\mathrm{N}$ ), nonpreferential to preferential residues ( $\mathrm{N}$ to $\mathrm{P}$ ), preferential to non-preferential residues $(\mathrm{P}$ to $\mathrm{N})$ and preferential to preferential residues (P to P) (Supplementary Table 5). Mutations at MHC contact position mutation were not examined because, as above, the numbers of these types of mutations were too small to achieve statistical significance. Mutations at TCR contact regions was also not examined because no residue preferences were found at these sites (see above).

We analyzed the proportion of these 4 types of mutation in the immunogenic and non-immunogenic datasets respectively. Interestingly, almost all (26 in 27) of the immunogenic neoantigens appear to be in the class of $\mathrm{N}$ to $\mathrm{P}$ preference (Supplementary Table 5; Fig. 4e). Statistical analysis shown that the $\mathrm{N}$ to $\mathrm{P}$ preference frequency of immunogenic neoantigens with mutations at anchor positions was significant higher (Fig. 4e, $\mathrm{p}<0.001$ ) than that for non-immunogenic neoantigens'. The other classes of preference did not show significant differences between the immunogenic and non-immunogenic neoantigens. Analyzing our results in more depth we found that one immunogenic neoantigen, MYADM, in the immunogenic peptide collection did not follow the $\mathrm{N}$ to $\mathrm{P}$ preference at its $\mathrm{C}$-terminal binding site for MHC. However, the original paper describing this immunogenic neoantigen showed that both the wild type and the mutant peptides of MYADM could elicit self T cell responses(49), suggesting that the wild type peptide, with its $\mathrm{C}$ terminal anchor, was binding the patient's MHC protein and immunogenic anyway. Thus, this neoantigen may not be a good target for further clinical trial and can be regarded as an exception in our study. the exception MYADM is not counted) of the immunogenic neoantigen mutations belong to the class of $\mathrm{N}$ to P preference and our detailed study of the KRAS G12D therapeutic neoantigen with the mutation 336 from the non-preferential residue $\mathrm{G}$ to the preferential residue $\mathrm{D}(\mathrm{N}$ to $\mathrm{P})$ is one of the clear examples. That is, neoantigens with mutations at anchor positions not belonging to the class of $\mathrm{N}$ to $\mathrm{P}$ preference

338 would be very unlikely to be immunogenic and our detailed study of the DPAGT1 V213L nonof the clear examples. Thus it could be a clear indicator for the exclusion of non-immunogenic 
neoantigen in the process of neoantigen prediction. From our NND dataset containing nonneoantigens with mutations at anchor positions were not $\mathrm{N}$ to $\mathrm{P}$ preference and could be eliminated during immunogenic neoantigen prediction with this indicator (Fig. 4f). Thus, we suggest that the processes we describe here could greatly help prediction of functional neoantigens for cancer immunotherapy.

\section{HLA-C*05:01 might be able to present KRAS G12D 10mer peptide}

349 Because the frequency of KRAS mutations in tumors is so high, mutations in this protein are attractive

350 targets for immunotherapy. So far, it is thought that targeting the KRAS G12D neoantigen would be useful only for patients expressing HLA-C*08:02. To expand use of the KRAS mutant we developed a strategy to screen other HLA-C alleles for their ability to bind KRAS G12D and thus have the potential to elicit antitumor responses.

Our strategy to identify new HLA candidates is divided into four steps. First, phylogenetic analysis was performed for the 15 different HLA-C alleles with relatively high frequency in human(50) (Supplementary Table 6; Supplementary Fig. 5). In the phylogenetic tree, three alleles $(\mathrm{C} * 07: 01$, $C^{*} 07: 02$ and $\left.C^{*} 17: 01\right)$ were distantly related to the reference allele, $C^{*} 08: 02$, and were excluded from further analyses (Supplementary Fig. 5). The relative distance of the remaining alleles to $\mathrm{C}^{*} 08: 02$ was calculated based on the phylogenetic tree. Secondly, their relative distance from $\mathrm{C}^{*}$ 08:02 was normalized as shown on the heatmap (Fig. 5a). This showed that $C * 05: 01$ is closely related to $C * 08: 02$ and might therefore be able to present the KRAS neoantigen. Third, sequence alignment combined with structure analyses was used to compare HLA-C*08:02 and other HLA-C alleles. Comparison of P3 and $\mathrm{P} \Omega$ binding motif of HLA-C alleles shown that $\mathrm{C}^{*} 05: 01$ is the only allele have the same sequence as C*08:02 at its P3 anchor binding motif (Supplementary Table 7; Supplementary Fig. 6). Moreover $\mathrm{C}^{*}$ 05:01 differed from $\mathrm{C}^{*}$ 08:02 by only two amino acids (at positions 77 and 80 on the $\alpha 1$ helix) at its $\mathrm{P} \Omega$ binding motif. Because of these observations we decided to test whether the changes would affect the ability of $C^{*}$ 05:01 to present the KRAS G12D peptide. We performed structural analyses based on our solved HLA-C*08:02 structure (C08-mut9m). 
370 Two of these are arginine amino acids which promote engagement of the peptide acidic residue (Fig.

3715 b; Supplementary Table 7) and thus may play important roles in presenting acidic P3 mutated

372 neoantigen. These three amino acids are the same in $C^{*} 05: 01$. The $P \Omega$ of peptide contacts amino acids $37377,80,84,143$ and 146 in $C^{*} 08: 02$ (Fig. 2c). Three of these (positions 84, 143, and 146) are invariable 374 in most HLA-C alleles (Supplementary Table 7). The two amino acids at positions 77 and 80 are also 375 the same in many of the HLA-C alleles but $C^{*} 05: 01$ and several other HLA-C alleles differ from $376 \mathrm{C}^{*} 08: 02$ by S77N and N80K. Amino acids at these positions pointed toward the TCR region and peptide respectively (Fig. 5c). However, these five residues may not affect the presentation of peptides 378 which use leucine as a c-terminal residue because, regardless of the amino acids they express at these 379 five positions, most of HLA-C alleles select leucine as a preferential residue at the P $\Omega$ position of 380 peptide (Supplementary Fig. 4). Thus, after these structural comparisons of HLA-C*05:01 and HLAC*08:02, we inferred that HLA-C*05:01 might also present mutant KRAS G12D peptide and therefore might be of potential use for T cell-based immunotherapy. We came to this conclusion in spite of the fact that peptide binding predictions suggest that HLA-C*05:01 would bind with only low affinity to both KRAS G12D 9mer and 10mer mutated peptides (Supplementary Table 2).

We next evaluated whether KRAS G12D can really be presented by C*05:01. We expressed C*05:01 heavy chain and $\beta 2 \mathrm{M}$ in E. coli and refolded them with 4 peptides: GADGVGKSA (mut9m, mutation site is indicated with the amino acid underlined); KRAS G12D 10mer peptide GADGVGKSAL (mut10m); wild type KRAS G12D 9mer peptide GAGGVGKSA (wt9m) and KRAS G12D 10mer peptide GAGGVGKSAL (wt10m). Only the mutant KRAS G12D 10mer peptide successfully refolded with HLA-C*05:01, suggesting that HLA-C*05:01 and HLA-C*08:02 are partially differ in their antigen presentation abilities. We solved the crystal structure of $C^{*}$ 05:01 in complex with the KRAS G12D 10mer peptide to a resolution of 1.9 angstroms (Supplementary Table 3). High quality resolution and electron density maps shown reliable placement of the 10mer peptide in $C^{*}$ 05:01 complex (Fig. 5d). The TCR contact positions P5, P6, P7 and P9 of the peptides are flexible and can't form stable conformations (Fig.5e). Nevertheless, the structure of the C05-mut10m closely resembles that of C08mut10m at the P1-P4 peptide region (Fig. 5e-g). Overlay of the C05-mut10m and C08-mut10m peptide regions shows that an exception occurs at the $\mathrm{P} 5-\mathrm{P} \Omega$ region of the peptides and position 77 and 80 of the HLA proteins (Fig. 5e). The peptide P8S positions in both the C05-mut10m and C08-mut10m act as axillary anchors. Differences also occur at the $\mathrm{P} 10 \mathrm{~L}$ position. Although $\mathrm{C} * 05$ and $\mathrm{C} * 08$ differ at 
position 77, the Asn77 of C05 and the Ser77 of C08 form hydrogen bonds with P10L in a similar way.

401 However, Asn80 from $C^{*} 05$ binds the oxygen of P10L while Lys80 from $C^{*} 08$ does not with the

402 oxygen of P10L shifting towards the a2 helix and thus losing one of the hydrogen bonds from Tyr84

403 (Fig. 5h). These results explain to some extent why the GADGVGKSA (mut9m) peptide did not

404 successfully refold with $C^{*} 05: 01$. The most likely cause is the change at position 80 , which will weaken the interaction between the 9 mer peptide and $C^{*} 05: 01$, thus reducing the likelihood of forming a stable complex. Excluding these differences, the peptide binding grooves of the HLA-C proteins had relatively conserved interactions with the KRAS G12D 10mer peptide. It is, however, possible that position 80 is located in the interface of the pMHC and T cell receptor, and there may change the morphology of the epitope and impact $\mathrm{T}$ cell selection.

In line with our hypothesis, the structure of HLA-C*05:01 in complex with KRAS G12D indicates that $C^{*} 05: 01$ can also present the KRAS G12D neoantigen but in a slightly different way than $C * 08: 02$ does. Therefore HLA-C*05:01 might also be a potential target for T cell-based cancer immunotherapy.

\section{An optimized procedure of neoepitope prediction}

Nowadays, most in silico neoepitope (neoantigen and corresponding HLAs) prediction methods have used neopeptide binding affinity to discover neoantigens for cancer immunotherapy. Although binding to MHC-I is currently the most popular computational filter for removing nonantigenic neopeptides, it still not robust enough to eliminate most of these candidates.

Our results emphasize the importance of anchor mutated neopeptides in cancer immunotherapy. We demonstrated that almost all of anchor mutated neoantigens which have induced autologous $\mathrm{T}$ cell response and are clinically effective have a mutation the demonstrates an $\mathrm{N}$ to $\mathrm{P}$ preference at anchor positions. Thus, we suggest an optimized procedure that will improve the accuracy of neoepitope prediction methods. The workflow of this procedure is shown in Fig. 6a. Tumor and normal samples are first obtained from cancer patient for genomic DNA and RNA sequencing and tumor infiltrating lymphocytes (TILs) isolation. Whole-exome sequencing analysis allows discovery of somatic mutations present in the tumor cells, but not in normal cells. In silico binding prediction is performed and can output a list of candidate peptides. The novelty of our procedure is in the following steps. 
for binding MHC), MHC contact position mutation (contact MHC but contribute much less) or TCR contact position mutation. Each type may use a different filtration strategy during neoantigen candidate prediction (Fig. 6b). This procedure may eliminate at least $42 \%$ of the anchor position mutated candidates. Also, the similarity score could be used to help judge the possibility that the TCR contact position mutated neopeptides will be immunogenic. These candidates can be further tested in vitro and later used in clinical trials. Meanwhile, a validated neoantigen can be used to identify other HLA alleles that might present it, as shown in our analyses of KRAS G12D and HLA-C*05:01.

\section{Discussion}

Recently $\mathrm{T}$ cells targeting tumor antigens, especially neoantigens, have proved to be one of the most promising new methods of treating cancers. However, few investigations have reported the structures of $\mathrm{T}$ cells reacting with cancer neoantigens. To fill this gap, here we solved the structure of one of the most successful clinical cancer immunotherapy neoantigens, KRAS with the G12D mutation, presented by HLA-C*0802. We found that the G12D mutation in these neoantigens (9mer and 10mer) drive intensive interactions to MHC via acting as an unconventional anchor residue. This observation of the mutation at the anchor position which cause the epitope to be immunogenic illustrates the complexity of neoantigens beyond the previous evidences that mutations which were solvent-exposed and therefore accessible to TCRs were more likely to be immunogenic. We next studied the structure of a non-immunogenic neoantigen, DPAGT1 V213L discovered from previously publication(19). Although

448 this peptide also had a mutation at the anchor position, it did not change the conformation of the TCR contact region. Thus different types of mutations in the anchor position of neoantigens would lead to different fates for immunogenicity.

To expand our findings more broadly and deeply we combined our structural work with the bioinformatics research in which the latter examined all available clinical data comparing cancer neopeptides that had proved to be immunogenic or non-immunogenic. Both types of mutated peptides from IND and NND were assigned to one of three different categories, those that contained mutations in anchor positions for MHC, or mutations in TCR contact regions or those that were mutated in contact regions with $\mathrm{MHC}$ that were not in anchor positions. Based on our results, mutations prefer to 
position) in IND. However, mutation frequency at anchor position did not show significant difference between IND and NND. And in fact, whether mutations in peptide anchor positions are important for the immunogenicity of the neoantigen was previously unclear. Different pieces of evidence led to different conclusions $(19,20,51,52)$. These assignments took into account the fact that the peptide anchor amino acids differ according to MHC allele with which they are engaged and the MHC anchor mutations were further categorized by whether or not the changed amino acids would improve binding of the peptide to $\mathrm{MHC}$ ( $\mathrm{N}$ to $\mathrm{P}$ changes), or reduce binding to $\mathrm{MHC}$ (P to $\mathrm{N}$ changes) or would not affect the ability of the peptide to bind MHC (N to N or P to P changes). Our analyses revealed a remarkable finding, for the immunogenic anchor position mutated neoantigens nearly all the changes fall into the non-preferential to preferential, $\mathrm{N}$ to $\mathrm{P}$, category (100\% if the exception one MYADM, which is always reactive no matter it has the mutation or not, was not counted). Thus this indicator are clear and practical for identifying anchor mutated neoantigens, called as "Non-preferential to Preferential Conversion (NPC)". The amino acid turning from non-preferential to preferential may create a new epitope which never seen by self-immune system. Given the fact that during the prediction of viral antigen, most amino acids in the peptide are different from self-peptide, such indicator above is not necessary for consideration. Hence, we believe NPC is an effective supplement indicator to eliminate non-immunogenic peptides from cancer mutaome in silico.

The way of neoantigen study and the rules for immunogenic neoantigens could be very different with normal antigens. Neoantigens are the altered-self between self-peptides and totally different foreign peptides. In conventional antigen immunogenicity research, how amino acids of multiple positions get together to interact with $\mathrm{MHC}$ and be recognized by TCR are main points. However, in neoantigen research, how one residue change could reshape the peptide interactions with $\mathrm{MHC}$ and/or reshape

480 TCR contact region as to break the immune tolerance are main points. Thus, mutation positions, mutation types, surrounding elements affecting mutations or affected by mutations and etc. should be paid more attentions. More specific bioinformatic strategies and more specific rules for immunogenic neoantigens are needed for research. In our study, certain rules were revealed only after large scale studies on human tumor mutant peptides and careful classification according to the mutation position in site is not predictive. Only if the mutation follows the NPC indicator does the change at an anchor position become valuable. 
488 On a related subject, here we tackled the issue of whether MHC alleles other than C*08:02 might be

489 useful in presenting the common KRAS G12D mutation as a neoantigen. We found that an MHC allele

490 that is closely related to $C^{*} 08: 02, C^{*} 05: 01$ might be such a possibility. Our structural work showed that

491 C*05:01 could indeed bind the KRAS G12D 10mer peptide and the configuration of the structure we

492 observed was quite similar to that of the same peptide bound to C*08:02. Thus, KRAS G12D mutation

493 could also be a good neoantigen in HLA-C*05:01 positive patients and the strategy we used to discover

494 this could be used to help enlarge knowledge of other suitable HLA alleles thus increasing the size of

495 the patient population in which such neoantigens could be used.

496 Our data suggest three possible immunogenic neoantigen models (Supplementary Fig. 7). Model A

497 represents the situation which peptide mutation occurs at TCR contact region and therefore appears

498 directly foreign to T cells. In this case, the neoantigens might be strongly immunogenic if the

499 corresponding wild type peptide is greatly dissimilar at the mutated site. Important differences might be amino acid properties such as hydrophobicity, charge and polarity. Model B represents the situation in which peptide mutation occurs at an MHC contact region (not including anchor sites). We found that mutations at these sites are less likely to render the neoantigen immunogenic. However, our curated data did show some bias for mutation at such regions, due to the relatively limited sample size available now for this category, the relationship for such between mutation and immunogenicity is not clear yet. Such mutants may still be immunogenic under certain conditions, if the mutant fits into an MHC pocket of dramatically changes the TCR contact surface of the pMHC complex. Model C represents the situation which the peptide mutation occurs at an MHC anchor position and increases MHC binding.

Overall, more structural and biochemical experiments, with inclusion of more MHC alleles are needed to improve bioanalytical predictive power in the important search for useful cancer neoantigens to treat these devastating diseases. We hope the methods proposed here will help guide such future studies.

\section{Materials and methods}

\section{Study design}


and non-immunogenic mutant peptides bound to MHCI. On the basis of these results, we also took bioinformatic analysis of our manually curated data form publications to see whether immunogenic peptides have a certain character for neoantigen prediction. Moreover, we also performed phylogenetic and structural analyses to extended neoantigen application.

\section{Protein Expression, refolding and purification}

522

The DNA encoding MHC heavy chain (HLA-C*08:02, HLA-C*05:01 and H-2 Kb) and light chain (human $\beta 2 \mathrm{M}$ and mouse $\beta 2 \mathrm{M}$ ) were synthesized (Idobio) and cloned into $\mathrm{pET}-22(\mathrm{~b})$ vector (Novagen). The vectors were transformed into the E. coli strain BL21 DE3 (Novagen). Transformants were selected from Lurian broth (LB) agar plates containing ampicillin. A single colony was selected and cultured in LB fluid medium with the antibiotics listed above at $37^{\circ} \mathrm{C}$. Upon reaching an optical density OD600 of 0.6 , expression was induced with the addition of $1 \mathrm{mM} \mathrm{IPTG.} \mathrm{Incubation} \mathrm{continued} \mathrm{at} 37^{\circ} \mathrm{C}$ for $5 \mathrm{~h}$. The cells were harvested by centrifugation and then resuspended in PBS buffer with $1 \mathrm{mM}$ PMSF at $4^{\circ} \mathrm{C}$. The cells were lysed, and the lysate was clarified by centrifugation at $10,000 \mathrm{~g}$ to collect inclusion bodies. Inclusion bodies were harvested and solubilized in $20 \mathrm{mM}$ Tris (Vetec) pH 8.0, $8 \mathrm{M}$ urea (Vetec), $1 \mathrm{mM}$ EDTA (BBI life sciences), $1 \mathrm{mM}$ DTT (Sinopharm chemical reagent) and $0.2 \mathrm{mM}$ PMSF (Sinopharm chemical reagent).

Refolding was performed in the presence of MHC heavy chain, $\beta 2 \mathrm{M}$ and peptides as described previously(53). Briefly, the resolubilized heavy chain and light chain in the presence of the corresponding peptide were added into 1 liter of refolding buffer. Refolding buffer contains $100 \mathrm{mM}$ Tris (pH 8.4), $0.5 \mathrm{mM}$ oxidized glutathione (BBI life sciences), $5 \mathrm{mM}$ reduced glutathione (BBI life sciences), 400mM L-arginine (Vetec), 2mM EDTA (BBI life sciences). After 48h refolding, the $1 \mathrm{~L}$ mixture dialyzed against 15 liters of $10 \mathrm{mM}$ Tris buffer $(\mathrm{pH} 8.0)$ at $4{ }^{\circ} \mathrm{C}$. for $24 \mathrm{~h}$.

Soluble proteins were first purified by anion exchange chromatography with Q Sepharose HP (GE Healthcare) column and Mono Q column (GE Healthcare). The purified protein sample was concentrated by tangential flow filtration using Amicon Ultra centrifugal filters (Merck). For desalination and purification, sample was loaded onto a Superdex 200 increase 10/300 GL column (GE Healthcare) for size exclusion chromatography. Chromatography performed with BioLogic DuoFlow system (Bio-rad) at a flow rate of $1 \mathrm{ml} / \mathrm{min}$. Peak analysis was performed using the ASTRA software 
package (BioLogic Chromatography Systems).

\section{Crystallization, data collection and processing}

548 Purified pMHC complex were concentrated to $10 \mathrm{mg} / \mathrm{ml}$ before the crystallization trials. For the HLA

549 complex, crystallizations were done by the sitting drop vapor diffusion technique at $4{ }^{\circ} \mathrm{C}$.

550 Crystallization screens were performed using a series of kits from Hampton Research. Single crystal of

551 C08-mut9m, C08-mut10m and C05-mut10m complex were obtained in the condition of $0.2 \mathrm{M}$

552 ammonium acetate, 0.1 M HEPES ( $\mathrm{pH} 7.5), 25 \% \mathrm{w} / \mathrm{v}$ polyethylene glycol 3,350. For the $\mathrm{H}-2 \mathrm{~Kb}$

553 complex, single crystal of $\mathrm{Kb}-8 \mathrm{mV}$ and $\mathrm{Kb}-8 \mathrm{~mL}$ complex were obtained when $4 \% \mathrm{v} / \mathrm{v}$ Tacsimate $(\mathrm{pH}$

$5547.0), 12 \% \mathrm{w} / \mathrm{v}$ Polyethylene glycol 3,350 was used as the reservoir buffer.

555 Before data collection, crystals were transferred to crystallization buffer containing 20\% (w/v) glycerol.

556 Next, the crystals were flash-cooled in liquid nitrogen immediately. The diffraction data were collected 557 at the Shanghai Synchrotron Radiation Facility (Shanghai, China) on beam line

558 BL17U1/BL18U1/BL19U1. The diffraction data were processed using the iMosflm program(54), and

559 data reduction was performed with Aimless Pointless in the CCP4 software suite(55). All structures

560 were determined by molecular replacement using the program Phaser(56). The models from the

561 molecular replacement were built using the COOT (Crystallographic Object-Oriented Toolkit)

$562 \operatorname{program}(57)$ and subsequently subjected to refinement using Phenix software(58). Data collection,

563 processing, and refinement statistics are summarized in (Supplementary Table 3). All the structural

564 figures were prepared using PyMOL program. The atomic coordinates and structure factors for the

565 reported crystal structures have been deposited on the Protein Data Bank (PDB;

566 http://www.rcsb.org/pdb/).

\section{Neoantigens and non-immunogenic neopeptides data collection and correction.}

569 Neoantigens and non-immunogenic neopeptides were manually curated with their corresponding HLA

570 alleles from 34 published literatures and recorded in immunogenic neoantigen dataset, IND and non-

571 immunogenic neopeptide dataset, NND.

572 To construct the immunogenic dataset IND, we collected 128 minimal peptides come from cancer 
573 somatic mutations and their corresponding HLA class I alleles that reported to elicit T cell response in

574 humans. Those tumor neopeptides with uncertain response or not provided with minimal sequence

575 were not collected in our dataset. Each entry includes following information: tumor type, gene symbol,

576 wild type/ mutant peptide sequence, wild type/mutant peptide predicted binding affinity (31), single

577 amino acid change, HLA allele, and PubMed ID from NCBI.

578 For non-immunogenic dataset NND, we collected 11739 peptides (not the minimal peptide) from

579 cancer somatic mutations and their corresponding HLA class I alleles that reported not elicit $\mathrm{T}$ cell

580 response in humans. Each entry includes following information: tumor type, gene symbol, wild type/

581 mutant peptide sequence, wild type/mutant peptide predicted binding affinity $(31)$, single amino acid

582 change, HLA allele, and PubMed ID from NCBI.

\section{Construction of non-immunogenic neopeptide dataset (NND)}

585 In order to generate minimal non-immunogenic neopeptide dataset, binding prediction was taken by

586 NetMHCpan 4.0 Server(40) based on the non-immunogenic neopeptide dataset (NND). Briefly,

587 different length of mutant peptides from NND were input in NetMHCpan 4.0 Server with custom

588 python scripts. The mutant peptides in 9- or 10-mer length with binding affinity under $500 \mathrm{nM}$ (IC50)

589 were considered to be binders and then recorded in the IND. Related peptide information was added to

590 each entry like IND dataset.

\section{Similarity comparison}

593 Similarity comparison of wild type and mutant amino acids were taken based on normalized

594 BLOSUM62 matrix as described previously(47). This matrix provides a value from 0 to 1 for representing the similarity of amino acid pairs (higher value indicates more similar). Tails of the violins to the range of the data were not trimmed.

\section{HLA present tendentiousness determination}



t-test.

described by Morten et al(59). Briefly, frequency of amino acid on distinct position was firstly computed using Hobonm 1 algorithm to decrease the weight of similar sequence before calculation.

It is worth to note that, in some cases, if the sample size is too large, it may include similar sequences, even duplicate sequences. Thus, clustering should be performed at first. For example, if one cluster include 10 similar sequences, the weight of every sequence in this cluster will be $1 / 10$. The similarity score was the maximum percent of the same amino acids between one current sequence and every sequence in one cluster, and the threshold of default similarity to decide whether current sequence belongs to a cluster is 0.63 .

In other cases, small sample related to amino acid depletion. Thus, we make use of pseudo counts to solve this problem. The formula to calculate the pseudo counts is

$$
g_{i}=\sum_{j} f_{j} \times q_{i \mid j}
$$

where $f_{j}$ is the real frequency of the amino acid $\mathrm{j}$ and $g_{i \mid j}$ is frequency of amino acid i replace amino acid $\mathrm{j}$ based on BLOSUM62 matrix(47).

After that, the estimated frequency is calculated according to equation

$$
P_{i}=\frac{\alpha \cdot f_{i}+\beta \cdot g_{i}}{\alpha+\beta}
$$

where $\alpha$ is the number of clusters subtracted by one. The default value $\beta$ is 200 .

Next, the half-bit $\log$ odds score is defined as

$$
W_{i, a}=2 \cdot \log _{2} \frac{p_{i, a}}{q_{a}}
$$

where $q_{a}$ is the background frequency of amino acids. In our case, an equiprobable background amino acid distribution is used.

The merged half-bits of one peptide is the summing half-bit score of amino acids in this peptide.

Statistical test of wild type peptides and mutant peptides merged half-bits from IND were tested using 
624 Nonameric peptide library obtained, peptide library sequence logo generation and HLA anchor position preferential determination

The nonameric peptide library of 30 HLA alleles was downloaded from IEDB(60). Sequence logos preferential amino acids at anchor position of each HLA alleles was applied to be above $10 \%(48)$ (include $10 \%$ ) based on the data from nonameric peptide library.

\section{HLA-C allele's frequency verification, phylogenetic analysis and heat map comparison}

HLA-C allele frequency was verified from Allele Frequency Net Database (AFND; http://www.allelefrequencies.net)(50). HLA-C alleles sequences were obtained from The IPD and IPDIMGT/HLA Database(62). Multiple sequence alignment was performed using MUSCLE (v3.8.31 for windows)(63). The input file and output file format were defaulted. We constructed phylogenetic trees using MrBayes (v3.2.5 for windows) by following the instruction of 'Quick Start Version' section in manual(64).

For display the allele similarities on the heat map, we read the phylogenetic tree using R package ape, and deleted some distantly related alleles $\left(C^{*} 07: 01, C^{*} 07: 02, C^{*} 17: 01\right)$. Similarity calculation was performed according to the distance of each HLA allele to $C^{*} 08: 02$. The similarity was normalized between $[0,1]$ and the heat map was plotted using $\mathrm{R}$ package heat map.

\section{Statistical analysis}

All data handling and nonlinear regression analyses were done using R, Microsoft Office Excel 2016 and GraphPad prism 6. P-values for difference were calculated using student's t test and Z-test.

\section{References and Notes}

1. M. D. Vesely, M. H. Kershaw, R. D. Schreiber, M. J. Smyth, Natural Innate and Adaptive Immunity 
to Cancer, Annu. Rev. Immunol. 29, 235-271 (2011).

2. Y. Sykulev, M. Joo, I. Vturina, T. J. Tsomides, H. N. Eisen, Evidence that a Single Peptide-MHC Complex on a Target Cell Can Elicit a Cytolytic T Cell Response, Immunity 4, 565-571 (1996).

3. P. C. Tumeh, C. L. Harview, J. H. Yearley, I. P. Shintaku, E. J. M. Taylor, L. Robert, B. Chmielowski, M. Spasic, G. Henry, V. Ciobanu, A. N. West, M. Carmona, C. Kivork, E. Seja, G. Cherry, A. J. Gutierrez, T. R. Grogan, C. Mateus, G. Tomasic, J. A. Glaspy, R. O. Emerson, H. Robins, R. H. Pierce, D. A. Elashoff, C. Robert, A. Ribas, PD-1 blockade induces responses by inhibiting adaptive immune resistance, Nature 515, 568-571 (2014).

4. T. N. Schumacher, R. D. Schreiber, Neoantigens in cancer immunotherapy, Science 348, 69-74 (2015).

5. N. A. Rizvi, M. D. Hellmann, A. Snyder, P. Kvistborg, V. Makarov, J. J. Havel, W. Lee, J. Yuan, P. Wong, T. S. Ho, M. L. Miller, N. Rekhtman, A. L. Moreira, F. Ibrahim, C. Bruggeman, B. Gasmi, R. Zappasodi, Y. Maeda, C. Sander, E. B. Garon, T. Merghoub, J. D. Wolchok, T. N. Schumacher, T. A. Chan, Mutational landscape determines sensitivity to PD-1 blockade in non-small cell lung cancer, Science 348, 124-128 (2015).

6. R.-F. Wang, H. Y. Wang, Immune targets and neoantigens for cancer immunotherapy and precision medicine, Cell Res. 27, 11-37 (2017).

7. M. Yadav, S. Jhunjhunwala, Q. T. Phung, P. Lupardus, J. Tanguay, S. Bumbaca, C. Franci, T. K. Cheung, J. Fritsche, T. Weinschenk, Z. Modrusan, I. Mellman, J. R. Lill, L. Delamarre, Predicting immunogenic tumour mutations by combining mass spectrometry and exome sequencing, Nature 515, 572-576 (2014).

8. P. G. Coulie, B. J. Van den Eynde, P. van der Bruggen, T. Boon, Tumour antigens recognized by T lymphocytes: at the core of cancer immunotherapy, Nat. Rev. Cancer 14, 135-146 (2014).

9. N. R. J. Gascoigne, V. Rybakin, O. Acuto, J. Brzostek, TCR Signal Strength and T Cell Development, Annu. Rev. Cell Dev. Biol. 32, 327-348 (2016).

10. E. Tran, P. F. Robbins, Y.-C. Lu, T. D. Prickett, J. J. Gartner, L. Jia, A. Pasetto, Z. Zheng, S. Ray, E. M. Groh, I. R. Kriley, S. A. Rosenberg, T-Cell Transfer Therapy Targeting Mutant KRAS in Cancer, N. Engl. J. Med. 375, 2255-2262 (2016).

11. P. A. Ott, Z. Hu, D. B. Keskin, S. A. Shukla, J. Sun, D. J. Bozym, W. Zhang, A. Luoma, A. GiobbieHurder, L. Peter, C. Chen, O. Olive, T. A. Carter, S. Li, D. J. Lieb, T. Eisenhaure, E. Gjini, J. Stevens, W. J. Lane, I. Javeri, K. Nellaiappan, A. M. Salazar, H. Daley, M. Seaman, E. I. Buchbinder, C. H. Yoon, M. Harden, N. Lennon, S. Gabriel, S. J. Rodig, D. H. Barouch, J. C. Aster, G. Getz, K. Wucherpfennig, D. Neuberg, J. Ritz, E. S. Lander, E. F. Fritsch, N. Hacohen, C. J. Wu, An immunogenic personal neoantigen vaccine for patients with melanoma, Nature 547, 217-221 (2017).

12. U. Sahin, E. Derhovanessian, M. Miller, B.-P. Kloke, P. Simon, M. Löwer, V. Bukur, A. D. Tadmor, U. Luxemburger, B. Schrörs, T. Omokoko, M. Vormehr, C. Albrecht, A. Paruzynski, A. N. Kuhn, J. Buck, 
S. Heesch, K. H. Schreeb, F. Müller, I. Ortseifer, I. Vogler, E. Godehardt, S. Attig, R. Rae, A. Breitkreuz, C. Tolliver, M. Suchan, G. Martic, A. Hohberger, P. Sorn, J. Diekmann, J. Ciesla, O. Waksmann, A.-K. Brück, M. Witt, M. Zillgen, A. Rothermel, B. Kasemann, D. Langer, S. Bolte, M. Diken, S. Kreiter, R. Nemecek, C. Gebhardt, S. Grabbe, C. Höller, J. Utikal, C. Huber, C. Loquai, Ö. Türeci, Personalized RNA mutanome vaccines mobilize poly-specific therapeutic immunity against cancer, Nature 547, 222-226 (2017).

13. P. F. Robbins, Y.-C. Lu, M. El-Gamil, Y. F. Li, C. Gross, J. Gartner, J. C. Lin, J. K. Teer, P. Cliften, E. Tycksen, Y. Samuels, S. A. Rosenberg, Mining exomic sequencing data to identify mutated antigens recognized by adoptively transferred tumor-reactive T cells, Nat. Med. 19, 747-752 (2013).

14. E. Tran, P. F. Robbins, S. A. Rosenberg, "Final common pathway" of human cancer immunotherapy: targeting random somatic mutations, Nat. Immunol. 18, 255-262 (2017).

15. T. Wolfel, M. Hauer, J. Schneider, M. Serrano, C. Wolfel, E. Klehmann-Hieb, E. De Plaen, T. Hankeln, K. Meyer zum Buschenfelde, D. Beach, A p16INK4a-insensitive CDK4 mutant targeted by cytolytic T lymphocytes in a human melanoma, Science 269, 1281 (1995).

16. H. Matsushita, M. D. Vesely, D. C. Koboldt, C. G. Rickert, R. Uppaluri, V. J. Magrini, C. D. Arthur, J. M. White, Y.-S. Chen, L. K. Shea, J. Hundal, M. C. Wendl, R. Demeter, T. Wylie, J. P. Allison, M. J. Smyth, L. J. Old, E. R. Mardis, R. D. Schreiber, Cancer exome analysis reveals a T-cell-dependent mechanism of cancer immunoediting, Nature 482, 400-404 (2012).

17. N. van Rooij, M. M. van Buuren, D. Philips, A. Velds, M. Toebes, B. Heemskerk, L. J. A. van Dijk, S. Behjati, H. Hilkmann, D. el Atmioui, M. Nieuwland, M. R. Stratton, R. M. Kerkhoven, C. Keşmir, J. B. Haanen, P. Kvistborg, T. N. Schumacher, Tumor Exome Analysis Reveals Neoantigen-Specific TCell Reactivity in an Ipilimumab-Responsive Melanoma, J. Clin. Oncol. 31, e439-e442 (2013).

18. P. F. Robbins, Y.-C. Lu, M. El-Gamil, Y. F. Li, C. Gross, J. Gartner, J. C. Lin, J. K. Teer, P. Cliften, E. Tycksen, Y. Samuels, S. A. Rosenberg, Mining exomic sequencing data to identify mutated antigens recognized by adoptively transferred tumor-reactive T cells, Nat. Med. 19, 747-752 (2013).

19. M. Yadav, S. Jhunjhunwala, Q. T. Phung, P. Lupardus, J. Tanguay, S. Bumbaca, C. Franci, T. K. Cheung, J. Fritsche, T. Weinschenk, Z. Modrusan, I. Mellman, J. R. Lill, L. Delamarre, Predicting immunogenic tumour mutations by combining mass spectrometry and exome sequencing, Nature 515, 572-576 (2014).

20. F. Duan, J. Duitama, S. Al Seesi, C. M. Ayres, S. A. Corcelli, A. P. Pawashe, T. Blanchard, D. McMahon, J. Sidney, A. Sette, B. M. Baker, I. I. Mandoiu, P. K. Srivastava, Genomic and bioinformatic profiling of mutational neoepitopes reveals new rules to predict anticancer immunogenicity, J. Exp. Med. 211, 2231-2248 (2014).

21. C. J. Cohen, J. J. Gartner, M. Horovitz-Fried, K. Shamalov, K. Trebska-McGowan, V. V. Bliskovsky, M. R. Parkhurst, C. Ankri, T. D. Prickett, J. S. Crystal, Y. F. Li, M. El-Gamil, S. A. Rosenberg, P. F. Robbins, Isolation of neoantigen-specific T cells from tumor and peripheral lymphocytes, J. Clin. Invest. 125, 3981-3991 (2015). 
22. B. M. Carreno, V. Magrini, M. Becker-Hapak, S. Kaabinejadian, J. Hundal, A. A. Petti, A. Ly, W.-R. Lie, W. H. Hildebrand, E. R. Mardis, G. P. Linette, A dendritic cell vaccine increases the breadth and diversity of melanoma neoantigen-specific T cells, Science 348, 803 (2015).

23. E. Tran, P. F. Robbins, Y.-C. Lu, T. D. Prickett, J. J. Gartner, L. Jia, A. Pasetto, Z. Zheng, S. Ray, E. M. Groh, I. R. Kriley, S. A. Rosenberg, T-Cell Transfer Therapy Targeting Mutant KRAS in Cancer, N. Engl. J. Med. 375, 2255-2262 (2016).

24. E. Stronen, M. Toebes, S. Kelderman, M. M. van Buuren, W. Yang, N. van Rooij, M. Donia, M.-L. Boschen, F. Lund-Johansen, J. Olweus, T. N. Schumacher, Targeting of cancer neoantigens with donor-derived T cell receptor repertoires, Science 352, 1337-1341 (2016).

25. V. Anagnostou, K. N. Smith, P. M. Forde, N. Niknafs, R. Bhattacharya, J. White, T. Zhang, V. Adleff, J. Phallen, N. Wali, C. Hruban, V. B. Guthrie, K. Rodgers, J. Naidoo, H. Kang, W. Sharfman, C. Georgiades, F. Verde, P. Illei, Q. K. Li, E. Gabrielson, M. V. Brock, C. A. Zahnow, S. B. Baylin, R. B. Scharpf, J. R. Brahmer, R. Karchin, D. M. Pardoll, V. E. Velculescu, Evolution of Neoantigen Landscape during Immune Checkpoint Blockade in Non-Small Cell Lung Cancer, Cancer Discov. 7, 264-276 (2017).

26. U. Sahin, E. Derhovanessian, M. Miller, B.-P. Kloke, P. Simon, M. Löwer, V. Bukur, A. D. Tadmor, U. Luxemburger, B. Schrörs, T. Omokoko, M. Vormehr, C. Albrecht, A. Paruzynski, A. N. Kuhn, J. Buck, S. Heesch, K. H. Schreeb, F. Müller, I. Ortseifer, I. Vogler, E. Godehardt, S. Attig, R. Rae, A. Breitkreuz, C. Tolliver, M. Suchan, G. Martic, A. Hohberger, P. Sorn, J. Diekmann, J. Ciesla, O. Waksmann, A.-K. Brück, M. Witt, M. Zillgen, A. Rothermel, B. Kasemann, D. Langer, S. Bolte, M. Diken, S. Kreiter, R. Nemecek, C. Gebhardt, S. Grabbe, C. Höller, J. Utikal, C. Huber, C. Loquai, Ö. Türeci, Personalized RNA mutanome vaccines mobilize poly-specific therapeutic immunity against cancer, Nature 547, 222-226 (2017).

27. N. Zacharakis, H. Chinnasamy, M. Black, H. Xu, Y.-C. Lu, Z. Zheng, A. Pasetto, M. Langhan, T. Shelton, T. Prickett, J. Gartner, L. Jia, K. Trebska-McGowan, R. P. Somerville, P. F. Robbins, S. A. Rosenberg, S. L. Goff, S. A. Feldman, Immune recognition of somatic mutations leading to complete durable regression in metastatic breast cancer, Nat. Med. 24, 724-730 (2018).

28. E. Stronen, M. Toebes, S. Kelderman, M. M. van Buuren, W. Yang, N. van Rooij, M. Donia, M.-L. Boschen, F. Lund-Johansen, J. Olweus, T. N. Schumacher, Targeting of cancer neoantigens with donor-derived T cell receptor repertoires, Science 352, 1337-1341 (2016).

29. M. Nielsen, C. Lundegaard, O. Lund, C. Keşmir, The role of the proteasome in generating cytotoxic T-cell epitopes: insights obtained from improved predictions of proteasomal cleavage, Immunogenetics 57, 33-41 (2005).

30. M. V. Larsen, C. Lundegaard, K. Lamberth, S. Buus, O. Lund, M. Nielsen, Large-scale validation of methods for cytotoxic T-lymphocyte epitope prediction, BMC Bioinformatics 8, 424 (2007).

31. M. Andreatta, M. Nielsen, Gapped sequence alignment using artificial neural networks: application to the MHC class I system, Bioinformatics 32, 511-517 (2016). 
32. M. M. Davis, P. Brodin, Rebooting Human Immunology, Annu. Rev. Immunol. 36, 843-864 (2018).

33. A. Vitiello, M. Zanetti, Neoantigen prediction and the need for validation, Nat. Biotechnol. 35, 815-817 (2017).

34. C. Kandoth, M. D. McLellan, F. Vandin, K. Ye, B. Niu, C. Lu, M. Xie, Q. Zhang, J. F. McMichael, M. A. Wyczalkowski, M. D. M. Leiserson, C. A. Miller, J. S. Welch, M. J. Walter, M. C. Wendl, T. J. Ley, R. K. Wilson, B. J. Raphael, L. Ding, Mutational landscape and significance across 12 major cancer types, Nature 502, 333-339 (2013).

35. C. H. June, Drugging the Undruggable Ras - Immunotherapy to the Rescue?, N. Engl. J. Med. 375, 2286-2289 (2016).

36. T. Kamisawa, L. D. Wood, T. Itoi, K. Takaori, Pancreatic cancer, The Lancet 388, 73-85 (2016).

37. F. F. González-Galarza, L. Y. C. Takeshita, E. J. M. Santos, F. Kempson, M. H. T. Maia, A. L. S. da Silva, A. L. T. e Silva, G. S. Ghattaoraya, A. Alfirevic, A. R. Jones, D. Middleton, Allele frequency net 2015 update: new features for HLA epitopes, KIR and disease and HLA adverse drug reaction associations, Nucleic Acids Res. 43, D784-D788 (2015).

38. T-Cell Transfer Therapy Targeting Mutant KRAS, N. Engl. J. Med. 376, e11 (2017).

39. M. Andreatta, M. Nielsen, Gapped sequence alignment using artificial neural networks: application to the MHC class I system, Bioinformatics 32, 511-517 (2016).

40. V. Jurtz, S. Paul, M. Andreatta, P. Marcatili, B. Peters, M. Nielsen, NetMHCpan-4.0: Improved Peptide-MHC Class I Interaction Predictions Integrating Eluted Ligand and Peptide Binding Affinity Data, J. Immunol. 199, 3360-3368 (2017).

41. M. Matsumura, D. Fremont, P. Peterson, I. Wilson, Emerging principles for the recognition of peptide antigens by MHC class I molecules, Science 257, 927-934 (1992).

42. H.-G. Rammensee, J. Bachmann, N. P. N. Emmerich, O. A. Bachor, S. Stevanović, SYFPEITHI: database for MHC ligands and peptide motifs, Immunogenetics 50, 213-219 (1999).

43. K. Christopher Garcia, J. J. Adams, D. Feng, L. K. Ely, The molecular basis of TCR germline bias for MHC is surprisingly simple, Nat. Immunol. 10, 143 (2009).

44. L. Yin, J. Scott-Browne, J. W. Kappler, L. Gapin, P. Marrack, T cells and their eons-old obsession with MHC, Immunol. Rev. 250, 49-60 (2012).

45. L. Yin, E. Huseby, J. Scott-Browne, K. Rubtsova, C. Pinilla, F. Crawford, P. Marrack, S. Dai, J. W. Kappler, A Single T Cell Receptor Bound to Major Histocompatibility Complex Class I and Class II Glycoproteins Reveals Switchable TCR Conformers, Immunity 35, 23-33 (2011).

46. J. J. Adams, S. Narayanan, M. E. Birnbaum, S. S. Sidhu, S. J. Blevins, M. H. Gee, L. V. Sibener, B. M. Baker, D. M. Kranz, K. C. Garcia, Structural interplay between germline interactions and adaptive recognition determines the bandwidth of TCR-peptide-MHC cross-reactivity, Nat. Immunol. 17, 87 
794

795

796

797

798

799

800

801

802

803

804

805

806

807

808

809

810

811

812

813

814

815

816

817

818

819

820

821

822

823

824

825

826

827

828

(2015).

47. S. Henikoff, J. G. Henikoff, Amino acid substitution matrices from protein blocks., Proc. Natl. Acad. Sci. 89, 10915-10919 (1992).

48. K. Falk, O. Rötzschke, S. Stevanovié, G. Jung, H.-G. Rammensee, Allele-specific motifs revealed by sequencing of self-peptides eluted from MHC molecules, Nature 351, 290-296 (1991).

49. N. McGranahan, A. J. S. Furness, R. Rosenthal, S. Ramskov, R. Lyngaa, S. K. Saini, M. JamalHanjani, G. A. Wilson, N. J. Birkbak, C. T. Hiley, T. B. K. Watkins, S. Shafi, N. Murugaesu, R. Mitter, A. U. Akarca, J. Linares, T. Marafioti, J. Y. Henry, E. M. Van Allen, D. Miao, B. Schilling, D. Schadendorf, L. A. Garraway, V. Makarov, N. A. Rizvi, A. Snyder, M. D. Hellmann, T. Merghoub, J. D. Wolchok, S. A. Shukla, C. J. Wu, K. S. Peggs, T. A. Chan, S. R. Hadrup, S. A. Quezada, C. Swanton, Clonal neoantigens elicit T cell immunoreactivity and sensitivity to immune checkpoint blockade, Science 351, 14631469 (2016).

50. F. F. González-Galarza, L. Y. C. Takeshita, E. J. M. Santos, F. Kempson, M. H. T. Maia, A. L. S. Da Silva, A. L. Teles E Silva, G. S. Ghattaoraya, A. Alfirevic, A. R. Jones, D. Middleton, Allele frequency net 2015 update: New features for HLA epitopes, KIR and disease and HLA adverse drug reaction associations, Nucleic Acids Res. 43, D784-D788 (2015).

51. E. F. Fritsch, M. Rajasagi, P. A. Ott, V. Brusic, N. Hacohen, C. J. Wu, HLA-Binding Properties of Tumor Neoepitopes in Humans, Cancer Immunol. Res. 2, 522-529 (2014).

52. M. Yarchoan, B. A. Johnson, E. R. Lutz, D. A. Laheru, E. M. Jaffee, Targeting neoantigens to augment antitumour immunity, Nat. Rev. Cancer 17, 209-222 (2017).

53. W. A. Macdonald, Z. Chen, S. Gras, J. K. Archbold, F. E. Tynan, C. S. Clements, M. Bharadwaj, L. Kjer-Nielsen, P. M. Saunders, M. C. J. Wilce, F. Crawford, B. Stadinsky, D. Jackson, A. G. Brooks, A. W. Purcell, J. W. Kappler, S. R. Burrows, J. Rossjohn, J. McCluskey, T Cell Allorecognition via Molecular Mimicry, Immunity 31, 897-908 (2009).

54. T. G. G. Battye, L. Kontogiannis, O. Johnson, H. R. Powell, A. G. W. Leslie, iMOSFLM : a new graphical interface for diffraction-image processing with MOSFLM, Acta Crystallogr. D Biol. Crystallogr. 67, 271-281 (2011).

55. M. D. Winn, C. C. Ballard, K. D. Cowtan, E. J. Dodson, P. Emsley, P. R. Evans, R. M. Keegan, E. B. Krissinel, A. G. W. Leslie, A. McCoy, S. J. McNicholas, G. N. Murshudov, N. S. Pannu, E. A. Potterton, H. R. Powell, R. J. Read, A. Vagin, K. S. Wilson, Overview of the CCP 4 suite and current developments, Acta Crystallogr. D Biol. Crystallogr. 67, 235-242 (2011).

56. A. J. McCoy, R. W. Grosse-Kunstleve, P. D. Adams, M. D. Winn, L. C. Storoni, R. J. Read, Phaser crystallographic software, J. Appl. Crystallogr. 40, 658-674 (2007).

57. P. Emsley, K. Cowtan, Coot : model-building tools for molecular graphics, Acta Crystallogr. D Biol. Crystallogr. 60, 2126-2132 (2004). 
829

830

831

832

833

834

835

836

837

838

839

840

841

842

843

844

845

846

847

848

849

850

851

852
58. P. D. Adams, R. W. Grosse-Kunstleve, L.-W. Hung, T. R. loerger, A. J. McCoy, N. W. Moriarty, R. J. Read, J. C. Sacchettini, N. K. Sauter, T. C. Terwilliger, PHENIX : building new software for automated crystallographic structure determination, Acta Crystallogr. D Biol. Crystallogr. 58, 1948-1954 (2002).

59. M. C. F. Thomsen, M. Nielsen, Seq2Logo: a method for construction and visualization of amino acid binding motifs and sequence profiles including sequence weighting, pseudo counts and twosided representation of amino acid enrichment and depletion, Nucleic Acids Res. 40, W281-W287 (2012).

60. R. Vita, J. A. Overton, J. A. Greenbaum, J. Ponomarenko, J. D. Clark, J. R. Cantrell, D. K. Wheeler, J. L. Gabbard, D. Hix, A. Sette, B. Peters, The immune epitope database (IEDB) 3.0, Nucleic Acids Res. 43, D405-D412 (2015).

61. M. C. F. Thomsen, M. Nielsen, Seq2Logo: a method for construction and visualization of amino acid binding motifs and sequence profiles including sequence weighting, pseudo counts and twosided representation of amino acid enrichment and depletion, Nucleic Acids Res. 40, W281-W287 (2012).

62. J. Robinson, J. A. Halliwell, J. D. Hayhurst, P. Flicek, P. Parham, S. G. E. Marsh, The IPD and IMGT/HLA database: allele variant databases, Nucleic Acids Res. 43, D423-D431 (2015).

63. R. C. Edgar, MUSCLE: multiple sequence alignment with high accuracy and high throughput, Nucleic Acids Res. 32, 1792-1797 (2004).

64. F. Ronquist, M. Teslenko, P. van der Mark, D. L. Ayres, A. Darling, S. Höhna, B. Larget, L. Liu, M. A. Suchard, J. P. Huelsenbeck, MrBayes 3.2: Efficient Bayesian Phylogenetic Inference and Model Choice Across a Large Model Space, Syst. Biol. 61, 539-542 (2012).

65. E. Paradis, J. Claude, K. Strimmer, APE: Analyses of Phylogenetics and Evolution in R language, Bioinformatics 20, 289-290 (2004). 
Figure 1. Structural comparison of HLA-C*08:02 in complex with therapeutic KRAS G12D

\section{9mer and 10mer neoantigens.}

857

858

859

860

861

862

863

a-b, Unambiguous 2Fo-Fc electron density maps of (a) KRAS G12D 9mer (GADGVGKSA, green) and (b)10mer (GADGVGKSAL, yellow) peptides from solved complex structures. The underlined amino acids represent the mutation in the peptides.

c, Overlay of the KRAS G12D 9mer and 10mer peptides.

d, Overlay of $\mathrm{C} \alpha$ traces of HLA-C*08:02 in complex with 9mer and 10mer peptides. These two structures show conformational differences at their peptide regions.

e, Sequence logo shows amino acid preference for 9mer peptide bounds to HLA-C*08:02. The peptide library obtained from IEDB, n=2321. Sequence logo was generated from Seq2Logo server.

f, Aromatic residues (green) from HLA-C*08:02 accommodating P1 and P2 residues of KRAS G12D 9mer peptide.

\section{Figure 2. Different presentation patterns between C08-mut9m and C08-mut10m}

a, Polar interactions between the HLA-C*08:02 molecule (grey) and 9mer (green), or HLA-C*08:02 interacts with 10mer (yellow) peptide at P1G and P2A positions.

b, KRAS G12D 9mer peptide interact with HLA-C*08:02 with the side chains P3D and P9A pointing down.

c, KRAS G12D 10mer peptide interact residues of HLA-C*08:02 with the side chains of P3D, P8S and P9A pointing down. 
877 a, Neoantigen predicted affinity ratio. Dots represents the ratio of predicted affinity of wild type peptide and predicted affinity of mutant peptide. All tested neoantigens were from the IND ( $\mathrm{n}=128)$.

879 Affinity prediction was performed using NetMHC 4.0pan. Orange dots $(n=26)$ represent the neoantigens with less than (or equal to) $500 \mathrm{nM}$ predicted affinity. Green dots $(\mathrm{n}=102)$ represent the neoantigens with more than $500 \mathrm{nM}$ predicted affinity. of 9 mer peptide. Positions of 9 mer peptide show on horizontal axis. on the three different classes: anchor position mutation, MHC contact position mutation and TCR contact position mutation. (immunogenic data, $\mathrm{n}=51$; non-immunogenic data, $\mathrm{n}=763$ ). contact position mutation and TCR contact position mutation) from IND and NND. The percentage of mutation distribution at anchor position did not shown significant difference between immunogenic and non-immunogenic data (ns, no significance). The frequency of mutation distribution at TCR contact region and $\mathrm{MHC}$ contact region shown significant difference $\left({ }^{*} \mathrm{p}<0.05, * * \mathrm{P}<0.01\right.$; immunogenic data, $\mathrm{n}=51$; non-immunogenic data, $\mathrm{n}=763$, Z-test). 


\section{Figure 4. Similarity and preference of data from IND and NND.}

896

897

898 counterparts to mutant peptides. The frequency between immunogenic and non-immunogenic data on

a-c, a, Similarity between wild type and mutant amino acids from immunogenic and non-immunogenic peptides. Normalized similarity score was calculated based on the BLOSUM62 matrix (ns, $\mathrm{p}=0.3077, \mathrm{n}$ $($ immunogenic data $)=128 ; \mathrm{n}($ non-immunogenic data $)=2883$, student $\mathrm{t}$-test $). \mathbf{b}$, Similarity between wild type and mutant amino acids from anchor position mutated immunogenic and non-immunogenic peptides $(* * \mathrm{p}=0.010, \mathrm{n}($ immunogenic data $)=29 ; \mathrm{n}($ non-immunogenic data $)=426$, student $\mathrm{t}$-test $) . \mathbf{c}$, Similarity of amino acids from 9mer TCR contact position mutated immunogenic and nonimmunogenic peptides $(* * \mathrm{p}<0.05, \mathrm{n}($ immunogenic data $)=40 ; \mathrm{n}($ non-immunogenic data $)=426$, student t-test).

d-f, $\mathbf{d}$, Comparation of HLA present tendentiousness between wild type and mutant 9 mer neoantigens from IND ( $n=69, * * p=0.0088$, student t-test); e, Comparation of HLA present tendentiousness between wild type and mutant 9 mer anchor mutated neoantigens from IND $(n=17, * * * * \mathrm{p}<0.0001$, student $\mathrm{t}$ test); $\mathbf{f}$, Comparation of HLA present tendentiousness between wild type and mutant 9mer TCR contact position mutated neoantigens from $\operatorname{IND}(n=39, p=0.9358$, student $t$-test).

g, Frequency of 4 different anchor mutation classes (non-preferential to non-preferential residues $(\mathrm{N}$ to $\mathrm{N}$ ), non-preferential to preferential residues ( $\mathrm{N}$ to $\mathrm{P}$ ), preferential to non-preferential residues (P to $\mathrm{N}$ ) and preferential to preferential residues (P to $\mathrm{P})$ ) of immunogenic data (from IND) and nonimmunogenic data (from NND). Each class is representing the preference alteration from wild type $\mathrm{N}$ to $\mathrm{P}$ class shows significant difference $(* * * \mathrm{p}<0.001, \mathrm{n}$ (immunogenic data) $=27 ; \mathrm{n}$ (nonimmunogenic data) $=425$, Z-test). 
Figure 5. HLA-C*05:01 is a potential allele to present KRAS G12D 10mer peptide. reference allele. spheres. spheres. solved C05-mut10m complex structures.

e, Overlay of the peptide region of C05-mut10m (cyan) and C08-mut10m (yellow) complexes. 


\section{HLA candidate discovery.}

a, Workflow of neoepitope discovery. Conventional procedures were indicated in solid box.

Propositional procedures in our study were indicated in dotted box. 
Supplementary Figure 2. Comparation of the mutation distribution of 10mer peptides from IND and NND.

(1/10) of 10mer peptide. Positions of 10mer peptide show on horizontal axis.

Supplementary Figure 3. Amino acids frequency of mutant peptides from IND and NND.

Supplementary Figure 4. 9mer peptides binding profile of 29 HLA alleles. 
Supplementary Figure 7. Different peptide-MHC models with their potential immunogenicity.

Model A demonstrates the peptides which have mutations at TCR contact positions and presented by similarities with the wild type. Model $\mathrm{C}$ demonstrates the peptides which have mutations at anchor anchor position. 
bioRxiv preprint doi: https://doi.org/10.1101/700732; this version posted August 23, 2019. The copyright holder for this preprint (which was not certified by peer review) is the author/funder. All rights reserved. No reuse allowed without permission. 
Figure 1

a

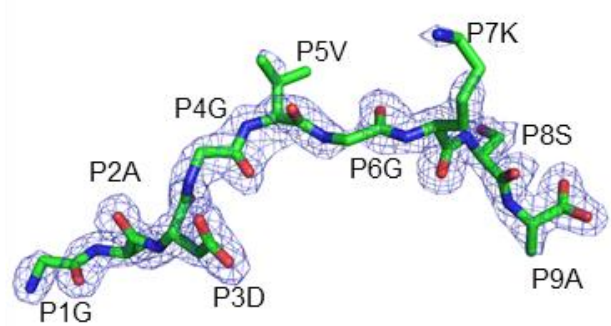

C

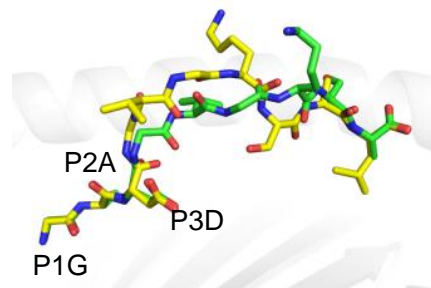

e

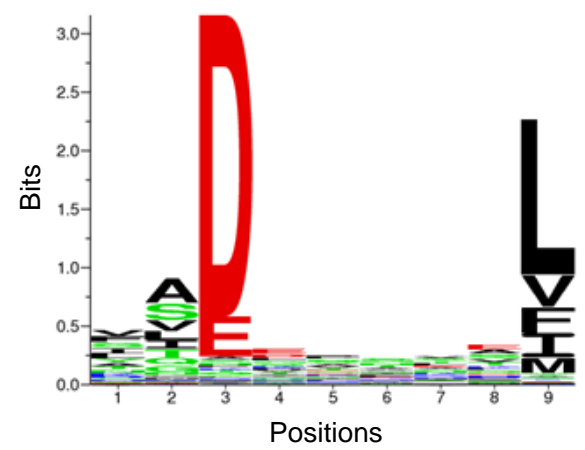

b

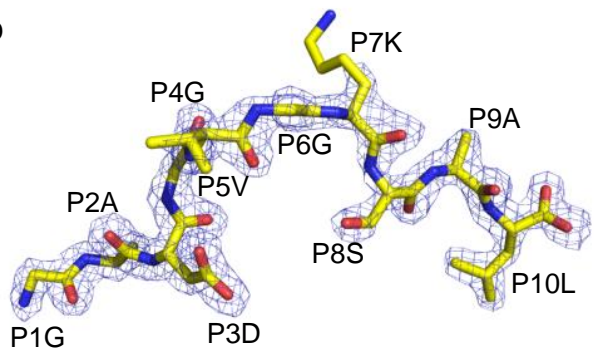

d

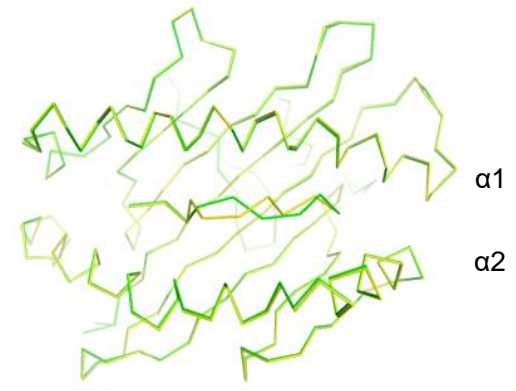

f

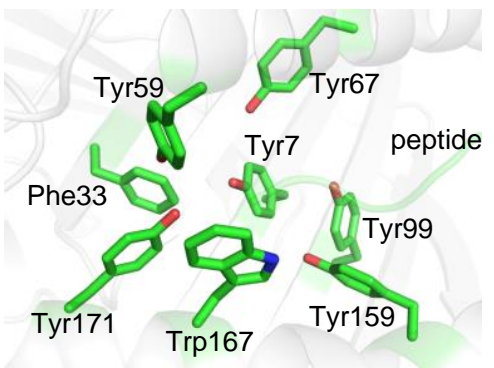


Figure 2

a

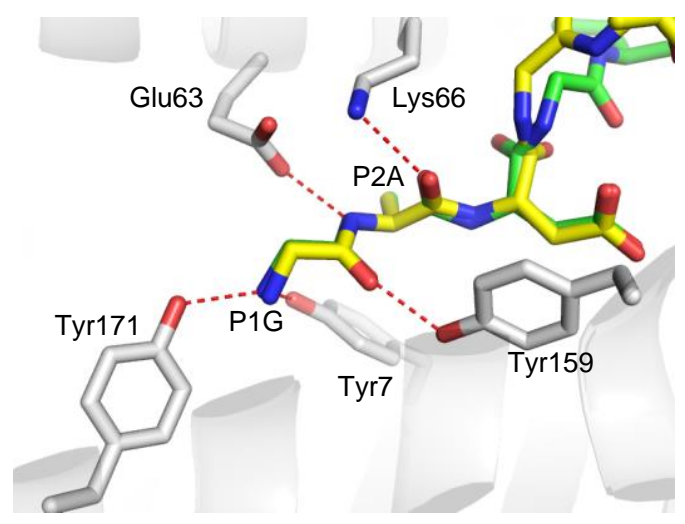

b

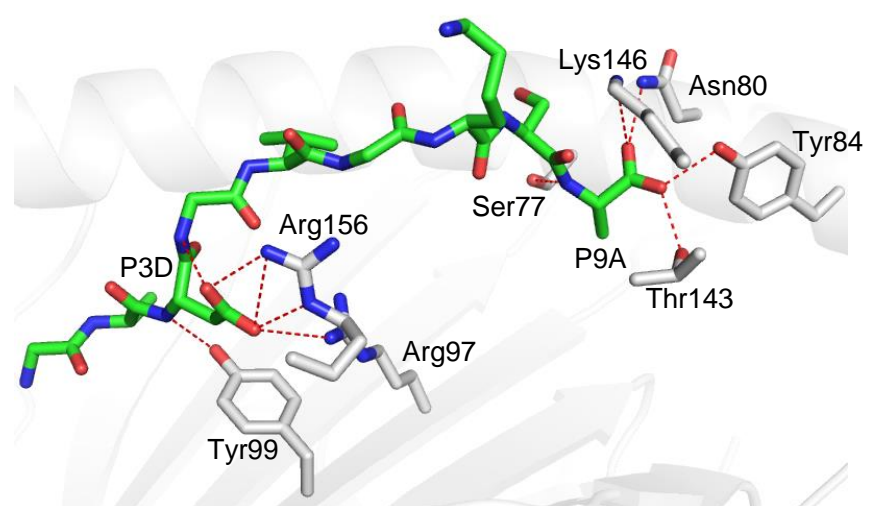

c

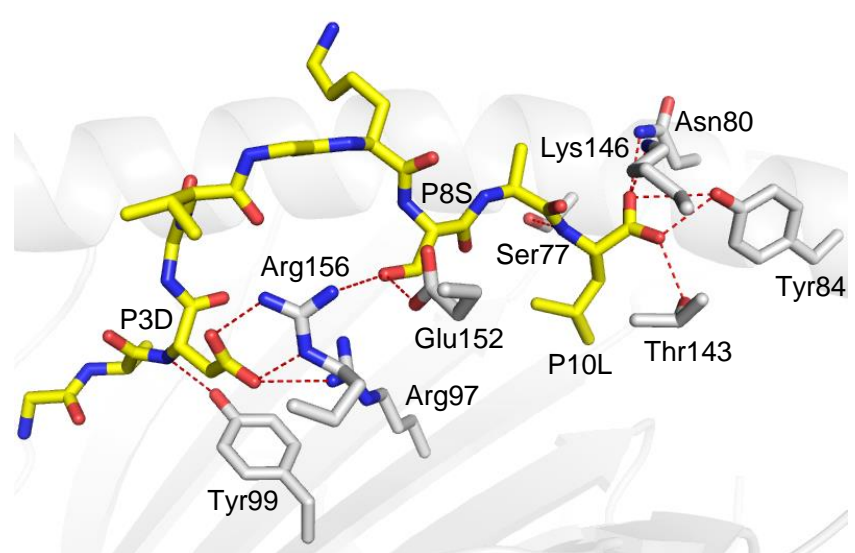


Figure 3

a

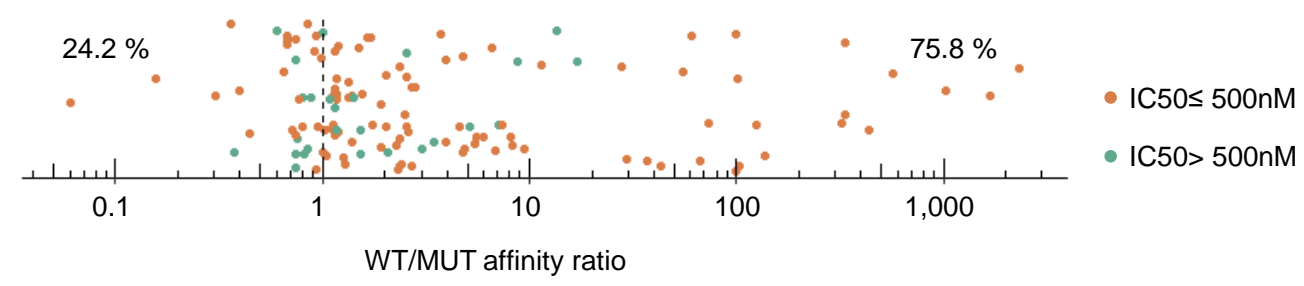

b

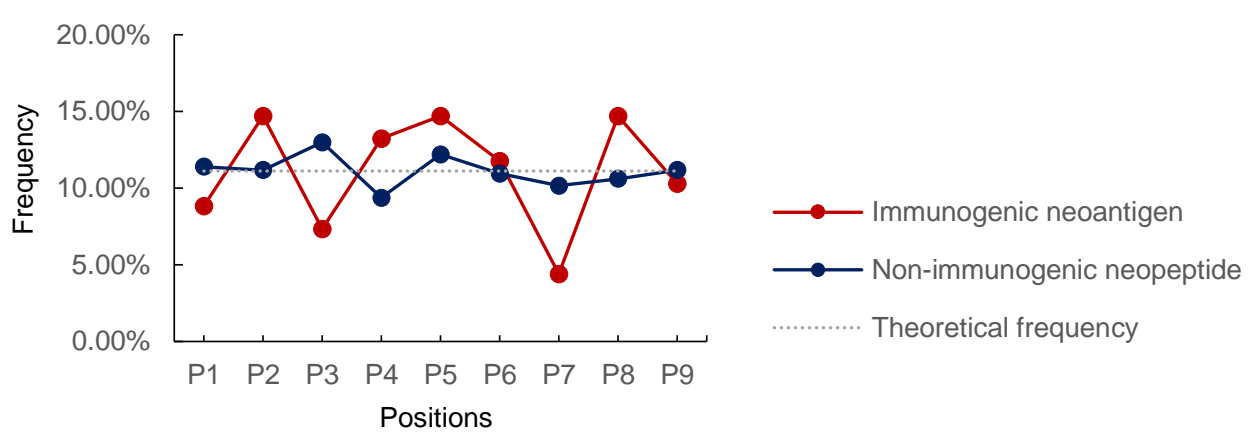

C
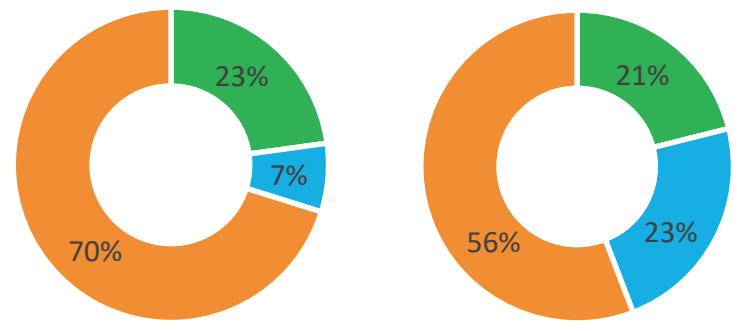

- Anchor position mutation

- MHC contact position mutation

Immunogenic neoantigens Non-immunogenic neopeptides

Immunogenic neoantigens Non-immunogenic neopeptides

- TCR contact position mutation

d

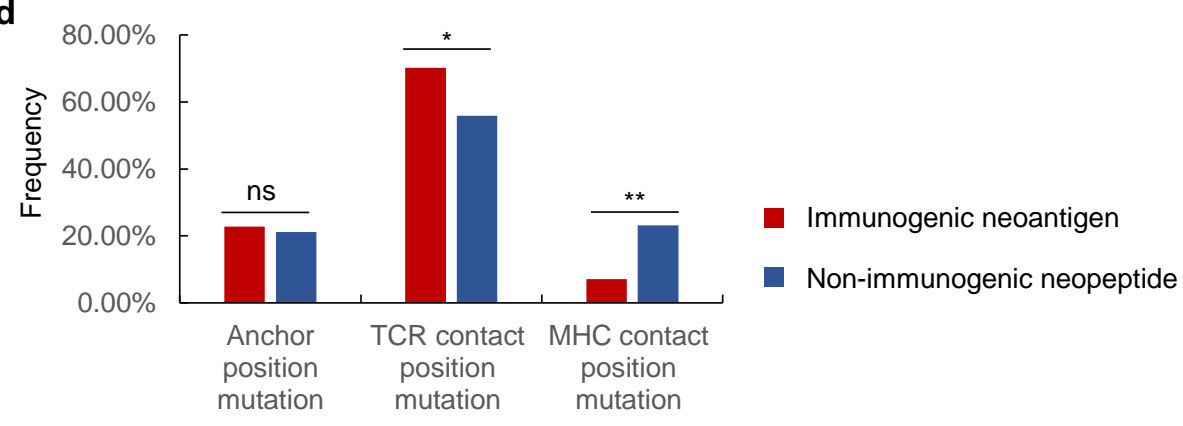




\section{Figure 4}

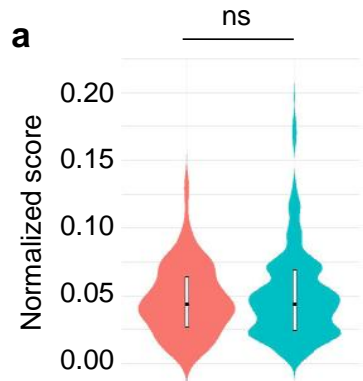

All mutations

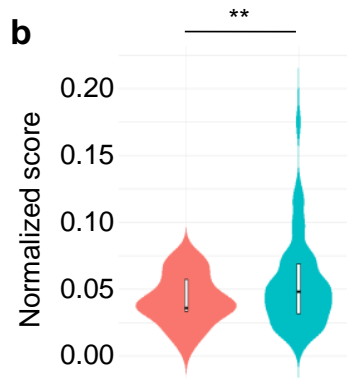

Anchor mutations

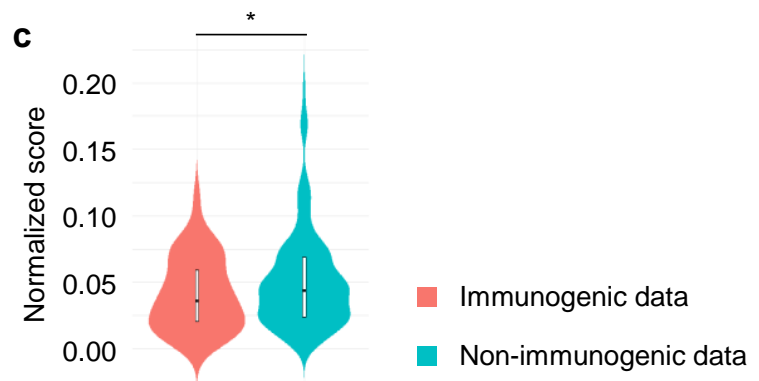

TCR contact position mutations
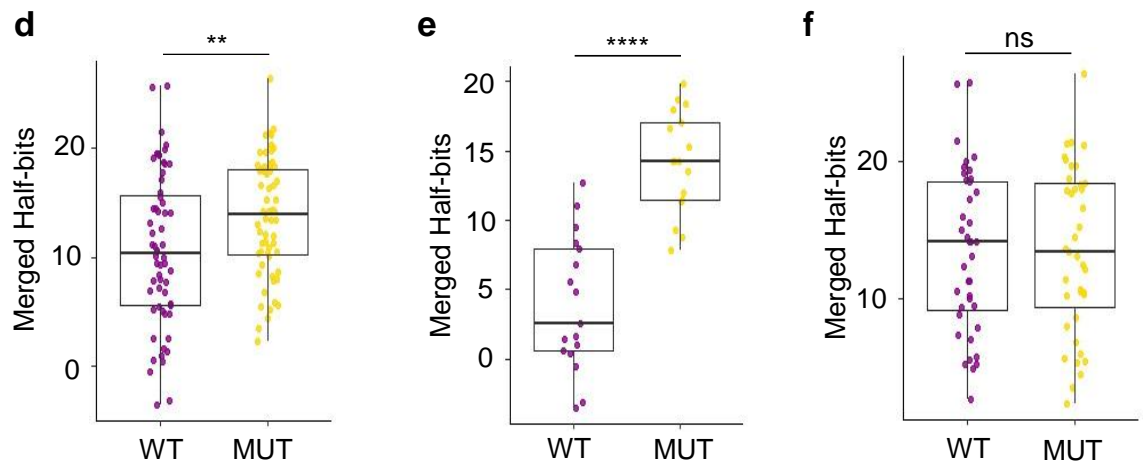

All 9mer immunogenic data

TCR contact position mutated data

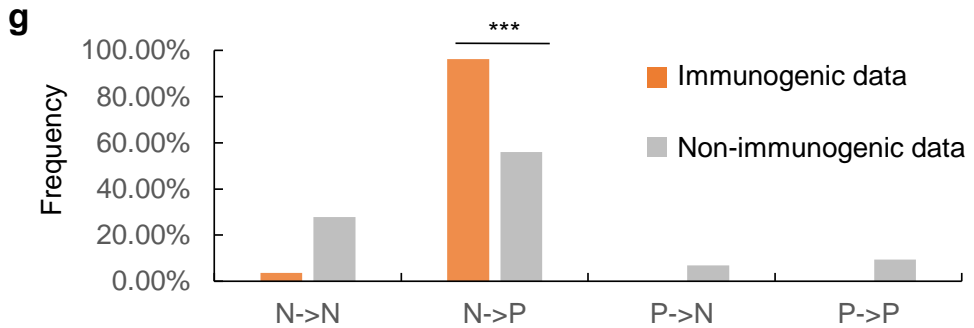

h

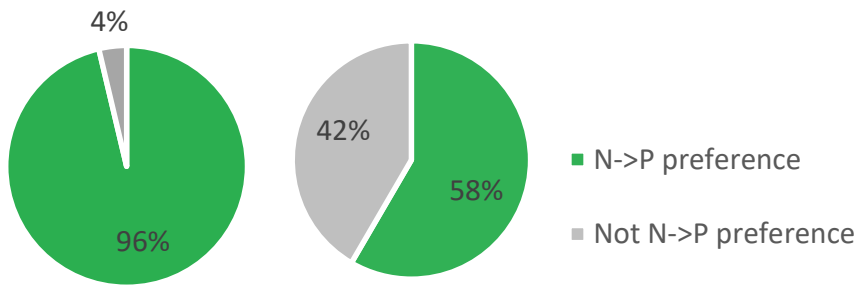

Immunogenic data Non-immunogenic data 


\section{Figure 5}

a

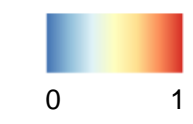

$C^{*} 08: 02$

$\mathrm{C}^{*}$ 05:01

$C^{*} 16: 01$

$C^{*} 12: 03$

$\mathrm{C}^{*} 06: 02$

$C^{*} 15: 02$

$\mathrm{C}^{*} 02: 02$

$C^{*} 14: 02$

C*01:02

C*03:04

$\mathrm{C}^{*} 03: 03$

$\mathrm{C}^{*}$ 04:01 b

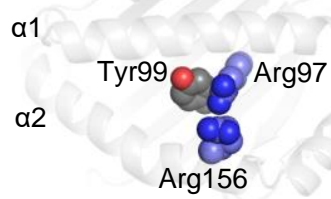

C

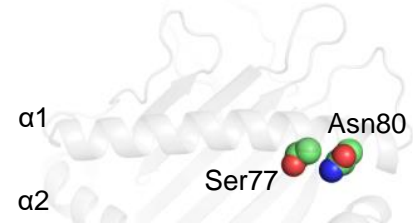

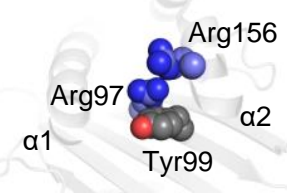

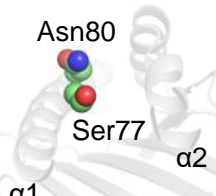

d

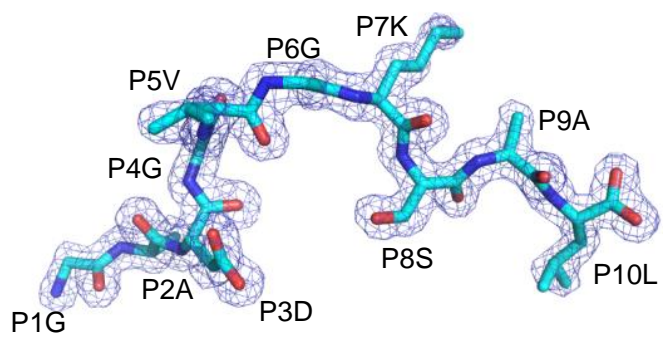

e

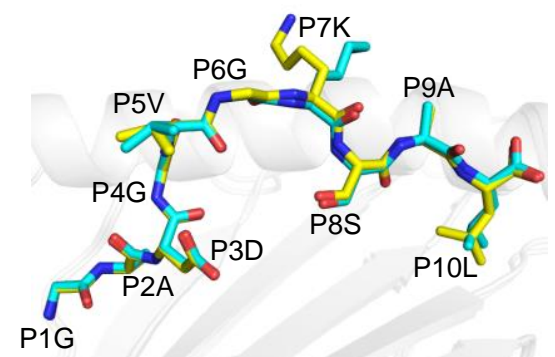

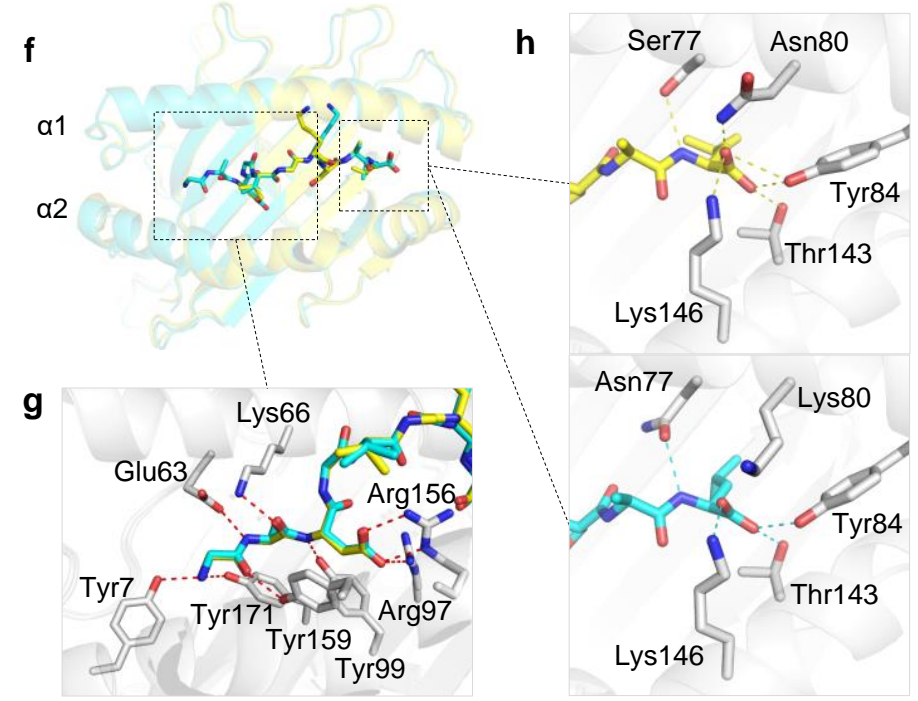




\section{Figure 6}

a

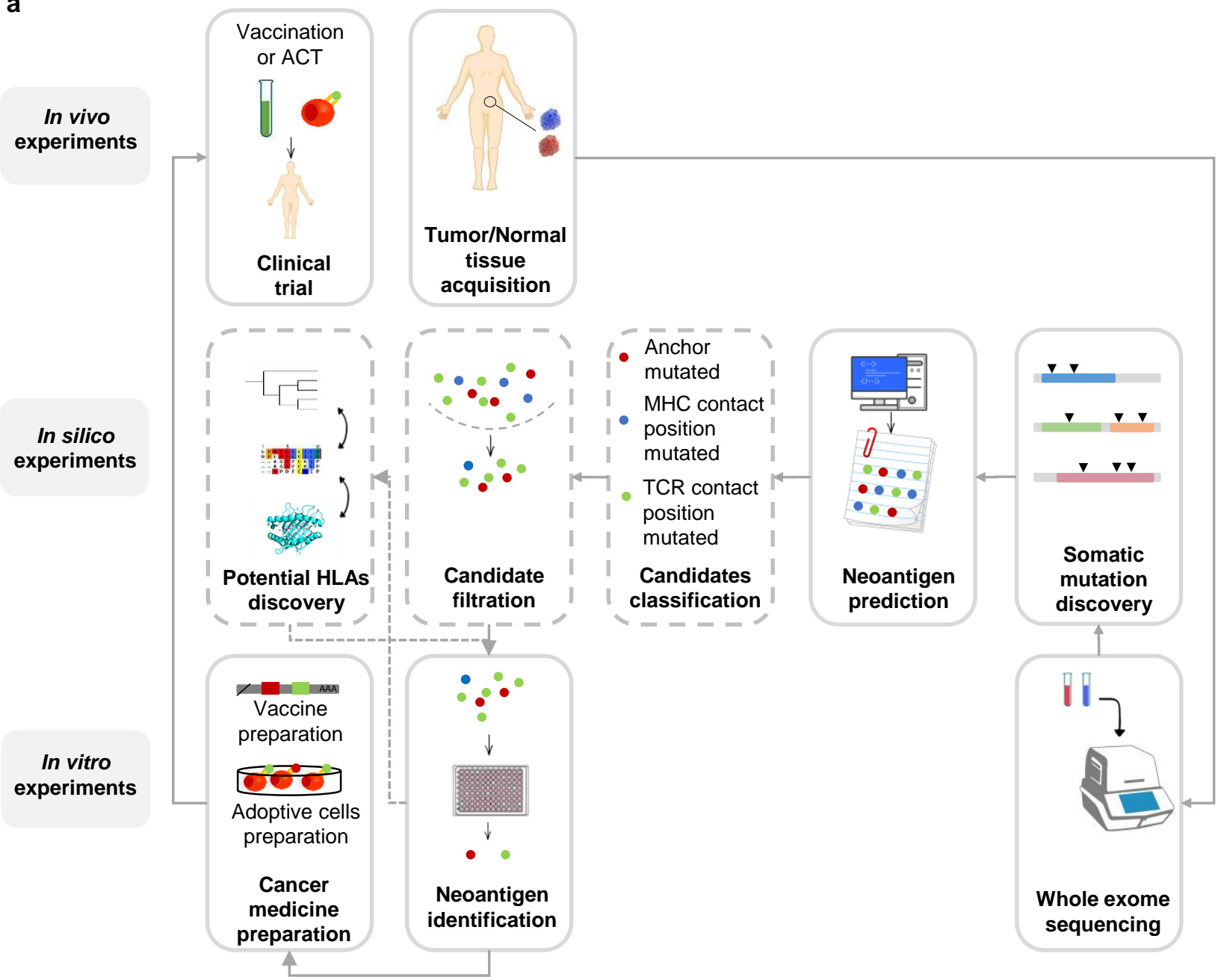

b

\begin{tabular}{|c|c|c|c|}
\hline & $\begin{array}{l}\text { Candidates } \\
\text { classification }\end{array}$ & $\begin{array}{l}\text { Candidates } \\
\text { filtration methods }\end{array}$ & $\begin{array}{l}\text { Candidates } \\
\text { eliminated }\end{array}$ \\
\hline $\begin{array}{l}\text { Anchor mutated } \\
\text { neopeptide }\end{array}$ & $\bullet \bullet \bullet$ & Preference selection & $\begin{array}{l}\bullet \bullet \\
{ }^{*} \text { Decrease } \sim 42 \%\end{array}$ \\
\hline $\begin{array}{l}\text { MHC contact position } \\
\text { mutated neopeptide }\end{array}$ & $\bullet \bullet$ & $\begin{array}{l}\text { Importance selection } \\
\text { Preference selection } \\
\text { Morphologic selection }\end{array}$ & $\bullet$ \\
\hline $\begin{array}{l}\text { TCR contact position } \\
\text { mutated neopeptide }\end{array}$ & $\bullet \bullet \bullet \bullet \bullet$ & $\begin{array}{l}\text { Dissimilarity selection } \\
\text { Morphologic selection }\end{array}$ & $\bullet \bullet \bullet \bullet$ \\
\hline
\end{tabular}

